\title{
Validation Analysis for the Calculation of a Turbulent Free Jet in Water using CFDS-FLOW3D and FLUENT (U)
}

by

Si Young Lee

and

Richard A. Dimenna

May 1995

Westinghouse Savannah River Company

Savannah River Technology Center

Aiken, SC29808

Prepared for the U.S. Department of Energy

Under Contract DE-AC09-89SR18035

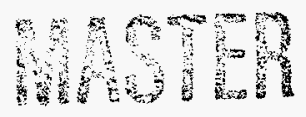




\section{DISCLAMMER}

This report was prepared as an account of work sponsored by an agency of the United States Government. Neither the United States Government nor any agency thereof, nor any of their employees, makes any warranty, express or implied, or assumes any legal liability or responsibility for the accuracy, completeness, or usefulness of any information, apparatus, product, or process disclosed, or represents that its use would not infringe privately owned rights. Referenice herein to any specific commercial product, process, or service by trade name, trademark, manufacturer, or otherwise does not necessarily constitute or imply its endorsement, recommendation, or favoring by the United States Government or any agency thereof. The views and opinions of authors expressed herein do not necessarily state or reflect those of the United States Government or any agency thereof.

This report has been reproduced directly from the best available copy.

Available to DOE and DOE contractors from the Office of Scientific and Technical Information, P.O. Box 62, Oak Ridge, TN 37831; prices available from (615) 576-8401.

Available to the public from the National Technical-Information Service, U.S. Department of Commerce, 5285 Port Royal Road, Springfield, VA 22161. 


\section{DISCLAIMER}

Portions of this document may be illegible in electronic image products. Images are produced from the best available original document. 
Validation Analysis for the Calculation of a

Turbulent Free Jet in Water

using CFDS-FLOW3D and FLUENT (U)

Si Young Lee

Richard A. Dimenna

Engineering Modeling and Simulations Group

Savannah River Technology Center

Approvals:

trac Hersel

Date: $5-4-25$

S. J. Hensel, Technical Reviewer

Gepeetrey

Date: $5-5.95$

J. R. Pelfrey, Manager, EMS

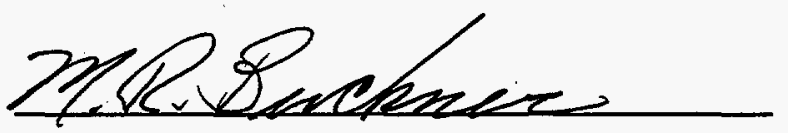

Date: $5-5-95$

M. R. Buckner, Manager, ATS

Mnchat PPoria

Date: $5 / 8 / 95$

M. R. Poirier, IWT, Customer Review 
Validation Analysis for the Calculation of a Turbulent Free Jet in Water using CFDS-FLOW3D and FLUENT (U)

\author{
S. Y. Lee \\ R. A. Dimenna \\ Engineering Modeling and Simulations Group
}

\begin{abstract}
The application of computational fluid dynamics methods to the analysis of mixing in the high level waste tanks at the Savannah River Site requires a demonstration that the computer codes can properly represent the behavior of fluids in the tanks. The motive force for mixing the tanks is a set of jet pumps taking suction from the tank fluid and discharging turbulent jets near the bottom of the tank. The work described here focuses on the free turbulent jet in water as the simplest case of jet behavior for which data could be found in the open literature. Calculations performed with both CFDS-FLOW3D and FLUENT were compared with data as well as classical jet theory. Results showed both codes agreed reasonably well with each other and with the data, but that results were sensitive to the computational mesh and, to a lesser degree, the selection of turbulence models.
\end{abstract}


Contents .

Abstract

Contents iii

Summary .iv

1.

2. Background. v

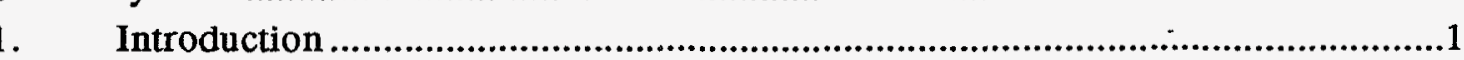

2.1 Conservation equations for turbulent flow...........................................6

2.2 Classical theory of the round (axisymmetric) free turbulent jet ..................7

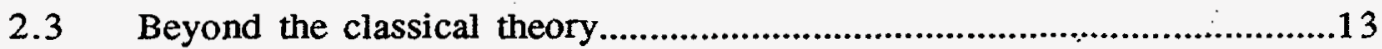

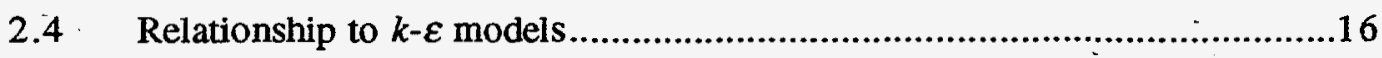

2.5 Reference data for this report............................................................18

3. Computational Code Analytical Models.........................................................19

3.1 Conservation equations...................................................................19

3.2 Significant physical observables..........................................................22

3.3 Options in the codes ..................................................................22

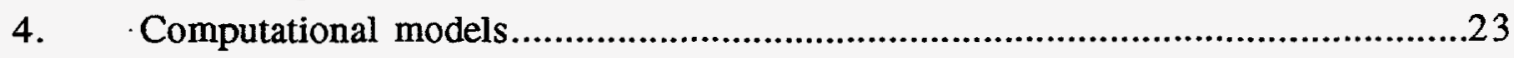

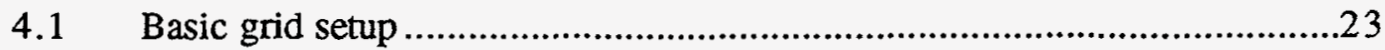

4.2 Parameters and boundary conditions .................................................28

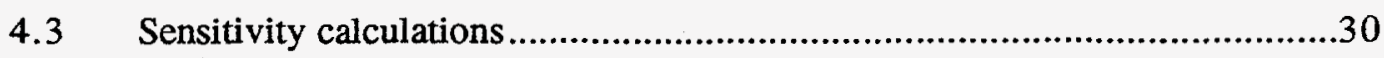

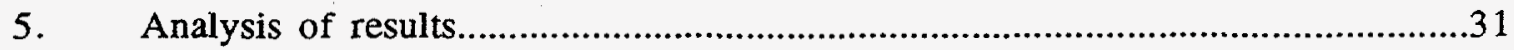

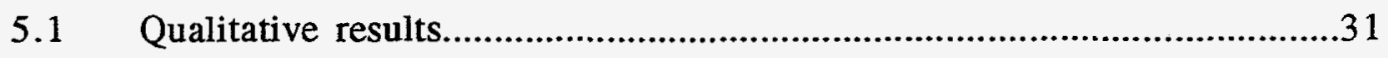

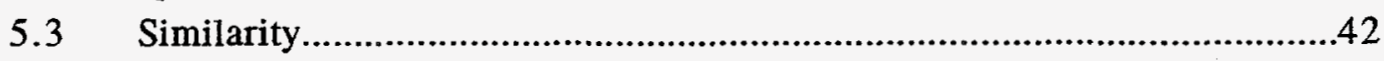

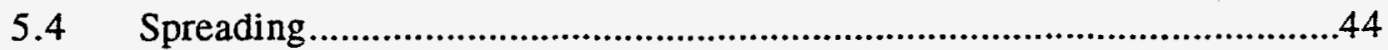

$5.5 \quad$ Turbulence parameters ................................................................45

$5.6 \quad$ Mesh sensitivity ..........................................................................50

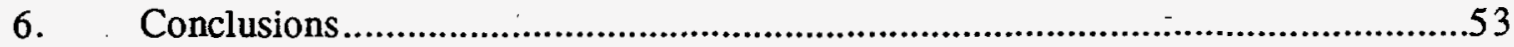

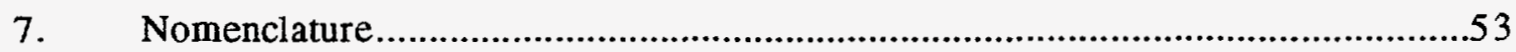

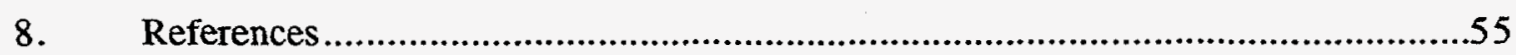

Appendix A 


\section{Summary}

The development of a computational mixing analysis capability at the Savannah River Site was initiated to provide operational guidance for the High Level Waste (HLW) storage and process tanks. Specifically, the High Level Waste Engineering (HLWE) = In-Tank Precipitation (ITP) organization requested information on how to operate the jet mixing pumps in Tank 48 to comply with tank temperature limits while maintaining adequate mixing behavior in the tank. Traditional phenomenological models and rules-of-thumb indicated that the pumps should be run continuously at full speed. The anticipated result of a more rigorous analysis with computational fluid dynamics (CFD) codes was that a different pump operational program could satisfy the mixing requirements in the tank with less total energy deposited into the tank. Such a finding would reduce, and perhaps eliminate, the need for additional heat removal capability to stay in compliance with operating temperature requirements. This report describes the work completed to establish the necessary computational capability, a demonstration that two CFD codes can properly represent the fundamental behavior of the turbulent liquid jets that mix the contents of the HLW tanks. The comparisons of liquid jet calculations with classical models and literature data will provide the fundamental code validation needed when these CFD codes are applied to specific tank mixing issues such as vortex formation or liquid climbing the tank walls.

Mixing the materials in the liquid region of the High Level Waste storage and process tanks will be accomplished through the use of submerged jet pumps that take suction from the tank fluid and discharge the fluid as a pair of high speed turbulent liquid jets in the vicinity of the tank bottom. The ability to observe or measure the actual performance of these pumps in providing adequate mixing is limited both by the size of the tanks and by the hazardous nature of the materials contained within. Therefore, computational fluid dynamics is seen as a way to evaluate the mixing behavior within the tanks as well as a way to interpret measurements of tank fluid parameters such as composition and temperature by relating the local measurements to the overall tank contents.

A necessary beginning to applying a computational technique to the tank mixing analysis is to demonstrate its applicability. The uncertainties associated with the materials contained in the waste tanks, and the lack of data specific to the performance of the pumps used in those tanks, requires that data used to validate the computational methods be taken from other sources, in particular, the open literature. The work described here includes a brief review of classical turbulent free jet theory, for this represents the only turbulent jet models that are amenable to an analytical solution. Simple relationships for volume and momentum flux, dissipation of the jet, and its rate of spreading are developed and used for comparison with calculated results from the two commercial CFD codes chosen for this work, CFDS- 
FLOW $3 D^{\circledR a}$ and FLUENT ${ }^{\circledR}$ b Extensive use is made of the classical mixing length theories, and their relationship to the more modern turbulence models used in the codes is discussed. In addition, more modern theories of turbulence structure are discussed insofar as they lend insight into the comparisons between the computational results and the classical theory.

Both computational codes employed here use the $k-\varepsilon$ model as their basic turbulence model. The simple nature of the turbulent free jet would indicate that this model should work fairly well in this study, and that proved to be the case. Comparisons with classical theory were good, and comparisons with data taken from the open literature were reasonable. Uncertainty data were not available to gauge the accuracy of the published data. Comparisons between the two codes were considered important because the ultimate application of these codes to tank mixing will be in a regime where virtually no data are available, either from the tanks themselves or in the open literature. In this situation, the only comparisons that will be available will be the results of another calculation.

The conclusions reached in this work are that the two codes selected for performing the mixing analysis treat the turbulent free water jet acceptably well. Calculations of axial velocity dissipation, axial velocity radial distributions (similarity profiles), jet spreading, momentum and volume flux profiles, velocity distributions perpendicular to the jet axis, and turbulence parameters show generally good agreement between the two codes, and where applicable, with classical jet theory. Where differences are apparent between the codes and classical turbulent jet theory, the codes appear to give the better results. This is to be expected, since the codes use a more modern and more detailed solution methodology, and they have the capacity to treat more of the details of the fluid motion than can be considered in the simplified classical theory. Nonetheless, differences were noted between the two codes. Therefore, results of calculations in more complex applications will have to be compared to each other carefully to discern the impact of different closure models, different noding schemes, different options selected, and perhaps different methods used to discretize the conservation equations.

\footnotetext{
a FLOW $3 D^{\circledR}$ is a registered trademark of AEA Industrial Technology, Harwell Laboratory, Oxfordshire, UK. The designation CFDS-FLOW3D is used here to distinguish the code from another with a similar name, FLOW-3D ${ }^{\circledR}$, marketed by Flow Science, Inc., Los Alamos, NM. b $^{\text {FLUENT }}{ }^{\circledR}$ is a registered trademark of FLUENT, Inc., Lebanon, NH.
} 


\section{Introduction}

The development of a computational mixing analysis capability at the Savannah River Site (SRS) was initiated to provide operational guidance for the High Level Waste (HLW) storage and process tanks. Specifically, the High Level Waste Engineering (HLWE) - In-Tank Precipitation (ITP) organization requested information on how to operate the jet mixing pumps in Tank 48 to comply with tank temperature limits while maintaining adequate mixing in the tank. Traditional phenomenological models and rules-of-thumb indicated that the pumps should be run continuously at full speed. The anticipated result of a more detailed analysis with computational fluid dynamics (CFD) codes was that a different pump operational program could optimize the mixing performance in the tank with the result of less total energy being deposited into the tank. This would be the case if a mixing analysis could show that all four pumps in Tank 48 did not have to run continuously, or that they could run at less than full speed. Such a finding would reduce, and perhaps eliminate, the need for additional heat removal capability to stay in compliance with operating temperature requirements. ${ }^{1}$

The purpose of this report is to establish the basis for using the results calculated by selected CFD codes. This basis is established by demonstrating that two CFD codes can properly represent the fundamental behavior of the turbulent liquid jets that mix the contents of the HLW tanks. Applications of the CFD codes to specific tank mixing issues such as vortex formation or liquid climbing the tank walls will then rely on the results of this report to validate the representation of the basic physics of the observed phenomena. This in turn will lend support to conclusions drawn from the calculated results.

Tank 48 is a Type IIIA tank, one of five types of large tanks used to store high level waste at SRS. From a liquid mixing point of view, it is typical in its geometry of the approximately 1million gallon tanks having a center column, but its contents are easier to characterize (in a computational sense) than those of the sludge tanks. Tank 48 contents are principally liquid with a varying concentration of suspended solids. The tank contents are mixed by four submerged pumps which rotate slowly while discharging opposing high speed jets of tank fluid. These jets are designed to maintain sufficient liquid motion to keep the contents homogeneous; thus ensuring that chemical reactions and washing operations are complete, and that solids remain uniformly distributed throughout the tank. The goal of the computational mixing analysis program is to determine the appropriate values for tank operational parameters which will optimize the ITP process. This will be accomplished by assessing their impact on tank mixing, heat generation, and entrainment of air into the liquid space. Similar questions have been raised about the performance of slurry pumps in suspending and mixing sludge in the Extended Sludge Processing (ESP) tanks such as Tank 42. Operational parameters beyond the initial set, or parameters specific to ESP tanks, may be identified in the course of completing the mixing analysis. 
The physical characteristics of the HLW storage and processing tanks at SRS makes a detailed analysis challenging. The tanks encompass a range of geometries, materials, and operational processes that extend beyond the current state of the art of mixing technology. The performance of any specific part of the system depends on incoming flow stream compositions as well as fluid behavior within the tank. Discharge stream composition must meet strict limits as well to satisfy the requirements imposed by downstream processes. A mixing analysis must be able to treat not only the effects of large physical dimensions of the tanks, but also the smaller scale effects such as jet nozzle diameter or specific injection sites for incoming streams. The operational parameters thought to be useful for optimizing process performance are those that directly affect mixing behavior, viz.:

1. Pump flow rate, including possible on-off cycles.

2. Pump elevation.

3. Pump rotation rate.

4. Batch size.

5. Tank liquid level.

The combination of these parameters, as well as the large physical size of the waste tanks and the hazardous nature of the contents makes a computational approach more attractive than an experimental one in terms of both time and cost to complete the work.

The method by which mixing performance or temperature compliance will be established within Tank 48 is as much a mixing issue as establishing flow patterns within the tank. The mixing behavior affects temperatures in the tank because energy is distributed in much the same fashion as momentum or material composition. Uniformity of tank temperature can be as much an issue as uniformity of material composition or uniformity of density in the form of build up in local regions of the tank. The interpretation of local temperature measurements as an indicator of bulk or mixed temperature is a mixing issue that can be addressed through the computational capabilities being developed.

The overall question of mixing performance in the HLW tanks was narrowed somewhat when three specific questions were posed by HLWE and IWT:

1. ... At what liquid level does a vortex formed from the pump operation begin to draw air into the liquid region of the tank?

2. At what liquid level does a pump discharge jet aimed parallel to or toward the liquid surface break the surface and begin to entrain air into the liquid region, the so-called "rooster tail" behavior?

3. At what tank liquid level does the liquid flow induced by the pumps climb the tank wall, break the liquid surface and begin to entrain air into the liquid region? 
These questions concerning ITP operations in Tank 48 are an integral part of the overall mixing issue, but they are less complex than the global mixing behavior. They involve the same basic driving phenomena that affect the overall mixing, so they represent a convenient set of issues that will be answered as interim goals in the analysis of tank mixing.

The questions concerning air entrainment as a function of liquid level can be treated without having to address the mixing issue per se. They are affected first by the behavior of the turbulent jet issuing from the pumps, and second by the free surface behavior at the interface between the liquid and air (or gas). Ultimately, the properties of the liquid region will affect the specific jet behavior, but at the outset the problem can be reduced to establishing the performance of the computational codes when calculating the characteristic behavior of turbulent free jets. The specific parameters to be assessed in conjunction with turbulent jet behavior will be discussed in detail in the next two sections. With that capability established, the calculations can proceed naturally to wall jets, rotating jets, free surface phenomena, wall interactions when the jet impinges on a vertical wall, and the interactions of flow patterns established by independent jets. Other phenomena needed for the tank mixing analysis such as particle suspension and setting rates, jet cleaning radius as sludge is suspended from the bottom of the tanks, or tank temperature behavior as the effects of energy transfers to and from the tank are included can be added incrementally.

The analytical approach to be used was determined by comparing the needs of the facilities with the advertised capabilities of computational fluid dynamics (CFD) packages available either commercially or from other government establishments. Using an existing code was considered more cost effective than writing a CFD code specifically for HLW tank analysis provided that the flexibility of commercial codes could satisfy the phenomenological needs. A review of a large number of available codes finally lead to a selection of two for this task, FLUENT and CFDS-FLOW3D. Both purport to be able to address the phenomena needed to perform our mixing analysis, and they appear to be more flexible than codes available from other government establishments. In addition, the user support available from a commercial organization is an advantage when developing a code model for a new and complex application.

The CFD codes will be applied to the mixing analysis task using a stepped approach designed to characterize code performance at the same time that the operational questions posed above are addressed. The procedure is described in detail in Reference 2, included with this report as Appendix A. Each of the HLWE / ITP questions posed above is characterized in terms of the fundamental phenomena needed to answer the question, and each of those phenomena is identified as a part of the computational analysis program. These parts are summarized as follows:

1. Jet behavior. Jet behavior is broken into three configurations, a turbulent free jet, a jet bounded by the bottom of the tank, and a surface jet. The first two configurations are to be treated as basic studies to characterize 
the performance of the CFD codes. They are not significantly affected by the operational parameters listed above, except that the proximity of the tank bottom is directly related to pump elevation for the bounded jet problem. The third configuration, the surface jet, is impacted by the location of the free surface, so it is a measure of the tendency of the jet to break the liquid surface. Therefore, this analysis addresses the "rooster tail" question. Since the free surface behavior is an integral part of the surface jet response, the treatment of the surface jet will be postponed until the next section of this analysis is completed, the vortex behavior.

2. Vortex behavior. Vortex behavior is directly related to free surface behavior and is thereby a significant test of a key feature of the CFD codes. The vortex analysis will address operational parameters such as pump speed, tank liquid level, and pump rotation. Since the codes are not expected to handle air entrainment properly, the objective of this analysis will be to determine the conditions for incipient air entrainment, but not to go beyond that point.

3. Wall climbing. Wall climbing behavior is the redirection of flow from an essentially horizontal orientation to a vertical orientation along the walls of the tank. This type of behavior is typical of calculations used in sales literature for CFD codes, so it is not expected to be a significant challenge for either code. It will be completed after the jet and vortex work described above.

4. Tank mixing. Tank mixing will present a significant challenge to the codes. The proper representation of mixing performance in the tanks will employ all of the capabilities described above, as well as all of the operational parameter variations and all of the physical parameter variations described in Reference 2. In addition to these many variables, the dimensions of the significant length scales in the tanks make the calculational representation difficult. Because mixing performance must be captured on the small length scale representative of the pump nozzle, about 1.5 inches, as well as on the large length scale of the tank, about 85 feet, the analysis will be broken into two regions. The region close to the pumps, the "near-region," will be affected principally by the jets of liquid as they emerge from the pumps, but not by distant effects such as the walls of the tank or the other pumps in the tank. The purpose of the near-region analysis is to calculate conditions at the edge of the region that can be used as boundary conditions for a second analysis of the rest of the tank. In this way, the scales affecting the analysis can be applied separately in the two regions and the grids used to represent the regions can be optimized individually. 
The tank analyses beyond the near-region will also be performed in two parts. The first will be to perform a 1/4-tank analysis in which a $90^{\circ}$ sector containing a single pump is modeled with symmetry boundary conditions. This will be used to assess the modeling technique as well as to characterize flow patterns and sensitivities within the tank. All of the operational parameters listed above will be included, some through the boundary conditions imposed from the near-region calculation, and some directly. The second part of the extended analysis will be to perform a 1/2-tank analysis in a manner similar to that used for the 1/4-tank analysis.

In summary, the purpose for this report is to establish the analysis and validation of the computations of the turbulent free jet to form the basis for all of the mixing work that is to follow. Since this jet behavior provides the basic mixing force that stirs the entire tank, it is important that the codes capture the free jet behavior adequately. The purpose for validating the behavior of the free jet in water is that so few data are available for validation in any other materials, with the possible exception of air.

It will be important to bear in mind the accuracy with which results can be calculated and compared with data. There are few data available for material properties of the contents of the waste tanks, and this will significantly impact the claimed accuracy of the calculations. In addition, there are few data concerning performance of the mixing operations in the tanks, so there isn't much to compare the calculations against. Therefore, small errors in the quantitative results of the calculations will probably not be detectable. The goal of the validation against water jets should be to identify potential sources of errors and ensure that they will remain small when the codes are extended to the waste tanks.

\section{Background}

The study of turbulent flow, jets, and wakes has been an area of vigorous research for well over 50 years. While progress has been made in understanding aspects of the flow and in developing models to represent turbulent flow phenomena, the details of turbulent flow which address local velocity fluctuations, the generation and dissipation of vortices, and the intermittency characteristic of the edges of turbulent shear flows are still unclear. The focus in this report on the behavior of a turbulent free jet narrows the breadth of the study significantly, but it is still affected by a large uncertainty as to the best treatment of shear phenomena near the edge of a turbulent jet.

Nearly all of the documented work on turbulent jets begins with the conservation equations for a Newtonian fluid, one in which the shear stress is related to the local velocity gradient by a constant viscosity. The turbulent momentum equations are developed by casting the components of the velocity vector in terms of a mean flow and a fluctuation about that mean, so

$$
u_{x}=\bar{u}_{x}+u_{x}^{\prime}
$$


where the mean value is denoted with an overscore and the fluctuating component with a prime.

\subsection{Conservation equations for turbulent flow}

The current analysis addresses the isothermal flow of a jet of water injected into a surrounding body of water at the same temperature. The two fundamental conservation equations describing this flow, in their general form, are: ${ }^{3}$

\section{Continuity}

$$
\frac{D \rho}{D t}=-\rho(\nabla \cdot u)
$$

Momentum

$$
\frac{\partial}{\partial t} \rho u+[\nabla \cdot \rho u u]=-\nabla p-[\nabla \cdot \tau]+\rho g
$$

This form of the momentum equation makes no assumptions about the nature of the fluid, in particular whether or not it is Newtonian. For the present analysis, the momentum equation can be simplified by the assumption of a constant density, $\rho$, and a constant viscosity, $\mu$. These simplifications result in the momentum equation being cast as the Navier-Stokes equation, $c$

$$
\rho \frac{D u}{D t}=-\nabla p+\mu \nabla^{2} u+\dot{\rho} g
$$

The conservation equations as expressed here represent each element of the velocity as a single value, say $u_{x}$. While this form of the conservation equations is general, an analysis of

${ }^{c}$ Bird, Stewart, and Lightfoot ${ }^{3}$ give one of the most readable developments of the momentum equation available, but they use a sign convention relating the shear stress to the velocity gradient that is different from most. In their notation, $\tau_{y x}=-\mu \partial u_{x} / \partial y$. Their reason for using this sign convention is that the shear stress is then easily interpreted as the transport of momentum across a constant $y$-plane and the sign is consistent with analogous relationships such as Fourier's law for one-dimensional heat conduction or Fick's law for one-dimensional mass diffusion. While some other authors use this same sign convention, ${ }^{4}$ most do not. The shear stress is more commonly defined as $\tau_{y x}=\mu \partial u_{x} / \partial y .5,6$ The developments in FLUENT and CFDS-FLOW3D use this more conventional approach. Because of the difference in sign conventions, the reader must be careful when reading the momentum equation when it is expressed in terms of the stress tensor. The differences disappear when the equation is expressed in terms of the viscosity, such as Eq. (2.4). 
turbulent flow is more easily accomplished when the velocity components are treated as the sum of a-mean value and a fluctuating component about that mean as shown in Equation (2.1). When this substitution is made in Equations (2.2) and (2.3) and the results time averaged over a time interval which is long compared to the time scale of the turbulent velocity fluctuations, the following forms for the continuity and momentum equations are obtained.

Continuity

$$
\nabla \cdot \overline{\boldsymbol{u}}=0
$$

Momentum

$$
\rho \frac{D \bar{u}}{D t}=-\nabla \bar{p}-\left[\nabla \cdot \bar{\tau}^{(1)}\right]-\left[\nabla \cdot \bar{\tau}^{(t)}\right]+\rho g
$$

These are now a set of conservation equations in terms of the mean flow with some differences in the momentum equation that result from the average of certain fluctuating terms. The term, $\left[\nabla \bullet \bar{\tau}^{(l)}\right]$, is analogous to the stress term in Equation (2.3) and represents the stresses associated with the mean velocity components of the flow. The additional term, $\left[\nabla \bullet \bar{\tau}^{(t)}\right]$, represents the stresses associated with the fluctuating components of the velocity. These are the so-called Reynolds stresses, and the model used to represent these Reynolds stresses characterizes the nature of the solution approach.

The validation work documented in this report focuses on the classical approach to turbulence modeling, since this represents about the only approach which can be used to find closed form solutions to the turbulent conservation equations. The following discussion will describe the general concepts and assumptions used in the classical turbulent jet theory employed in this analysis. It will then discuss some extensions to that work from the more recent literature, as well as more modern transport models used to represent turbulent flow behavior. The more modern approaches require numerical solutions to the governing equations, so they require code-to-code comparisons rather than comparisons with analytical solutions. Finally, the data to be used for comparisons with FLUENT and CFDS-FLOW3D will be presented.

\subsection{Classical theory of the round (axisymmetric) free turbulent jet}

Experimental and theoretical analysis of the axisymmetric free turbulent jet has been documented for many years, so much so that much of the work has found its way into textbooks which themselves are considered by many to be classics. ${ }^{7,8}$ One of the most detailed expositions is that given by Hinze, ${ }^{7}$ and it is that reference which forms the basis for 

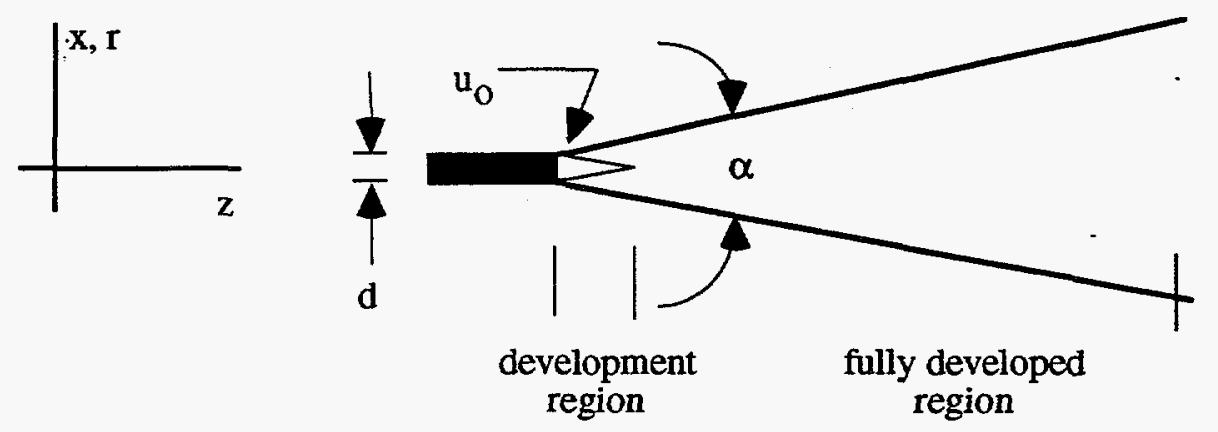

Figure 1. Turbulent free jet

the present discussion on round free turbulent jets. The general behavior envisioned for the jet is as shown in Figure 1.

The development region of the jet is that distance from the nozzle over which the axial velocity along the centerline of the jet is essentially unchanged from the discharge velocity at the nozzle. This region persists for about five nozzle diameters from the nozzle and gradually dissipates as the velocity profile across the cross-section of the jet forms a similarity profile. This formation of a similarity profile describing the axially directed velocity as a function of perpendicular distance from the jet axis is a key assumption in the classical jet theory. The development of these profiles is well established experimentally.

The second key assumption employed in the classical theory is conservation of momentum flux in the turbulent jet. This assumption arises from an assumption that there are no external forces acting on the jet, therefore the momentum must be conserved. The assumption of constant momentum flux in the jet has received both support $7,8,9$ and criticism, ${ }^{10}$ but its usefulness for describing the general nature of the jet is well established.

Hinze's development of the axisymmetric jet relationships begins with a statement of the conservation equations for a time independent, axisymmetric flow with constant density. The axisymmetric condition is applied by setting all derivatives with respect to the angular coordinate, $\theta$; to zero, as well as setting the value of $u_{\theta}$ to zero. Expanding Equation (2.5) into component notation and applying these simplifying assumption results in the following continuity equation for the round jet.

$$
\frac{\partial}{\partial z}\left(u_{s}+\bar{u}_{z}\right)+\frac{1}{r} \frac{\partial}{\partial r}\left(r \bar{u}_{r}\right)=0
$$

$u_{s}$ is the velocity of the ambient fluid, taken to be zero in our application. Therefore, the continuity equation becomes 


$$
\frac{\partial}{\partial z}\left(\bar{u}_{z}\right)+\frac{1}{r} \frac{\partial}{\partial r}\left(r \bar{u}_{r}\right)=0
$$

The same assumptions are applied to the momentum equation, Equation (2.6), which when cast in component form yields the following result for the axial direction momentum balance.

$$
\bar{u}_{z} \frac{\partial \bar{u}_{z}}{\partial z}+\bar{u}_{r} \frac{\partial \bar{u}_{z}}{\partial r}=\frac{1}{r} \frac{\partial}{\partial r} r\left(-\overline{u_{r}^{\prime} u_{z}^{\prime}}\right)
$$

In addition to the assumptions stated above, this expression for the axial momentum balance has assumed that the viscous terms in Equation (2.6) can be neglected compared to the Reynolds stress terms. Therefore, the viscosity does not appear in Equation (2.8). Since the ambient flow is constant (zero in this case), the axial pressure gradient in the ambient fluid is zero. The boundary layer assumptions applied to the momentum equation then give a zero axial pressure gradient in the turbulent region as well.

Some limitations to the applicability of Equation (2.8) are expected because the viscous terms were eliminated. In general, it is applicable to high Reynolds number flows where the Reynolds stress terms are large compared to the viscous terms. The correct behavior near a wall or far from the origin of the jet would not be captured because turbulent fluctuations there would be significantly diminished compared to the fully developed similarity region and viscous effects would be more important. Nonetheless, the approximation is useful for describing the general behavior of the jet in the fully developed region.

Experimental observations have shown that the axial velocity profiles perpendicular to the axis of the jet are similar when scaled by a characteristic velocity and length. Using the issuing jet velocity, $u_{o}$ as the velocity scale and the diameter of the jet, $d$, as the length scale, the similarity relationships are expressed in the following general form.

$$
\begin{aligned}
& \bar{u}_{z}=u_{o}\left(\frac{z+a}{d}\right)^{P} f\left(\xi_{2}\right) \\
& \bar{u}_{r}=u_{o}\left(\frac{z+a}{d}\right)^{r} g\left(\xi_{2}\right) \\
& \overline{-u_{r}^{\prime} u_{z}^{\prime}}=u_{o}^{2}\left(\frac{z+a}{d}\right)^{s_{21}} h\left(\xi_{2}\right)
\end{aligned}
$$

where

$$
\xi_{2}=\frac{r / d}{[(z+a) / d]^{q}}
$$


The position $z=-a$ is the "origin of similarity," that location at which the two edges of the spreading jet would come together were they to be extended backward beyond the jet orifice.

The similarity expressions are substituted into the mass and momentum balance equations, the integrated momentum balance over the range of $r$ from 0 to $\infty$ is taken as constant, and the quantity $-u_{r}^{\prime} u_{z}^{\prime} / \bar{u}_{z}^{2}$ is assumed to be independent of $z$ to evaluate the coefficients in the similarity relationships. The result is the following values for the coefficients.

$$
p=-1 \quad q=1 \quad r=-1 \quad s_{21}=-2
$$

Then the similarity relationships become

$$
\begin{aligned}
& \bar{u}_{z}=u_{o}\left(\frac{z+a}{d}\right)^{-1} f\left(\xi_{2}\right) \\
& \bar{u}_{r}=u_{o}\left(\frac{z+a}{d}\right)^{-1} g\left(\xi_{2}\right) \\
& \overline{-u_{r}^{\prime} u_{z}^{\prime}}=u_{o}^{2}\left(\frac{z+a}{d}\right)^{-2} h\left(\xi_{2}\right)
\end{aligned}
$$

and

$$
\xi_{2}=\frac{r / d}{[(z+a) / d]}=\frac{r}{z+a}
$$

When the turbulent shear stress is evaluated in terms of an eddy viscosity, $v_{t}$, and a mean velocity gradient,

$$
\overline{-u_{r}^{\prime} u_{z}^{\prime}}=v_{t} \partial \bar{u}_{z} / \partial r
$$

the above similarity relationships result in an expression for $v_{t}$ that is also independent of $z$.

When the similarity relationships, Equations (2.9), are substituted into the momentum equation, Equation (2.8), the resulting expression is

$$
-\left(f^{2}+\xi_{2} f \frac{d f}{d \xi_{2}}\right)+g \frac{d f}{d \xi_{2}}=\frac{1}{\xi_{2}} \frac{d}{d \xi_{2}}\left(\xi_{2} h\right)
$$

With the Boussinesq approximation that $v_{t}$ is constant and with the relationship 


$$
v_{t}=\frac{-\overline{u_{r}^{\prime} u_{z}^{\prime}}}{\partial \bar{u}_{z} / \partial r}
$$

the $\bar{u}_{z}$ velocity profile is evaluated from Equation (2.10) as

$$
\frac{\bar{u}_{z}}{\bar{u}_{z, \max }}=\left[1+\frac{\bar{u}_{z, \max }(z+a)}{8 v_{t}} \xi_{2}^{2}\right]^{-2}
$$

Since $\bar{u}_{z, \max }$ is the value of $u_{z}$ along the jet axis, its value can be determined from Equation (2-9a) by setting $\xi_{2}=0$ or $f\left(\xi_{2}\right)=1$, thereby evaluating Equation (2-9a) on the jet axis.

$$
\begin{aligned}
& \left.\bar{u}_{z}\right|_{\xi_{2}=0}=\bar{u}_{z, \max }=u_{o}\left(\frac{z+a}{d}\right)^{-1} \\
& \bar{u}_{z, \max }=\frac{u_{o} d}{z+a}
\end{aligned}
$$

With this simplification, Equation (2.11) is more conveniently written as

$$
\frac{\bar{u}_{z}}{\bar{u}_{z, \max }}=\left[1+\frac{u_{o} d}{8 v_{t}} \xi_{2}^{2}\right]^{-2}
$$

Together, Equations (2.12) and (2.13) provide relationships against which the calculated turbulent jet behavior can be compared. Equation (2.12) shows the decay of the axial jet velocity as distance from the orifice increases, while Equation (2.13) gives the axial velocity distribution in a plane perpendicular to the axis. Both of these expressions are applicable only in the fully developed region of the jet, which begins at about five diameters from the jet orifice.

The value of $v_{t}$ can be estimated from the Prandtl mixing length theory. ${ }^{7,8}$ That theory indicates that the eddy viscosity can be represented by a length scale multiplied by a suitable velocity. A characteristic length scale is the half width of the jet, the so-called mixing region, and is denoted $\ell_{m}$. A characteristic velocity can be determined from a mean velocity gradient and the length scale. A mean velocity gradient is estimated by dividing the axial velocity by the width of the mixing region, giving

$$
\frac{\partial \bar{u}_{z}}{\partial r} \approx \frac{\bar{u}_{z, \max }}{\ell_{m}}
$$

Multiplying this mean gradient by the mixing length, $\ell_{m}$, will then give a characteristic velocity for mixing. The characteristic length, $\ell_{m}$, can be evaluated in terms of the observed spreading angle of the jet, $\alpha$, and the axial distance from the orifice. 


$$
\ell_{m}=(z+a) \tan \left(\frac{1}{2} \alpha\right)
$$

Then $v_{t}$ is

$$
\begin{aligned}
v_{t} & \approx \ell_{m}^{2}\left|\frac{\partial \bar{u}_{z}}{\partial r}\right| \\
& \approx \tan \left(\frac{1}{2} \alpha\right) u_{o} d
\end{aligned}
$$

An approximate relationship is indicated in Equation (2.14) and the expression for a mean velocity gradient because these are only estimates for the eddy viscosity and should not be considered precise evaluations.

The rate of volume entrainment in the turbulent jet is another characteristic parameter that can be used to compare the computational results with the classical behavior. If the volume flux is taken as $\Phi$, and the volume flux issuing from the nozzle as $\Phi_{O}$, the issuing volume flux can be expressed as

$$
\Phi_{0}=(\pi / 4) d^{2} u_{o}
$$

and the volume contained within the jet can be determined from

$$
\frac{\Phi}{\Phi_{0}}=\frac{8}{d^{2}} \int_{0}^{\infty} r \frac{\bar{u}_{z}}{u_{0}} d r
$$

With Equations (2.9a) and (2.9d), this results in an entrainment relationship of

$$
\frac{\Phi}{\Phi_{0}}=\frac{A(z+a)}{d}
$$

where $A$ is a constant evaluated as

$$
A=8 \int_{0}^{\infty} \xi_{2} f\left(\xi_{2}\right) d \xi_{2}
$$

It must be noted that this expression, according to Hinze, is not a reliable measure of the turbulent jet because it fails to account for the intermittent character of the turbulent and laminar flow patterns at the edges of the jet when the integral is extended to infinity. Therefore, it should be considered a qualitative measure of the jet, at best. Nonetheless, the 
general linear dependence on $z$ is approximately correct and can be observed in the comparisons with the computational models.

Additional relationships associated with the classical round turbulent jet model described by Hinze that can be compared with the computational solutions are the shear stress distribution in the jet,

$$
\frac{\sigma_{r z}}{\rho \bar{u}_{z, \max }^{2}}=-\frac{1}{2} \xi_{2}\left[1+\frac{\bar{u}_{z, \max }(z+a)}{8 v_{t}} \xi_{2}^{2}\right]^{-3}
$$

which shows a maximum magnitude for the turbulent shear stress, $\sigma_{r z}$, at

$$
\left(\xi_{2}\right)_{o p t}=\left[\frac{8 v_{t}}{5 \bar{u}_{z, \max }(z+a)}\right]^{1 / 2}
$$

for $v_{t}$ constant, and the half width of the similarity profile,

$$
\left(\xi_{2}\right)_{1 / 2}=\left[\frac{8(\sqrt{2}-1) v_{t}}{\bar{u}_{z, \max }(z+a)}\right]^{1 / 2}
$$

Other researchers have described the same general behavior for jets as that described above taken from Hinze. While it is impossible to list them all here, useful review articles describing some more recent work that show general support for the classical behavior of turbulent jets include those by List ${ }^{11}$ and Rajaratnam. ${ }^{12}$

\subsection{Beyond the classical theory}

The jet theory described in Section 2.2 is admittedly simple. Several key assumptions allowed important simplifications to the conservation equations, including similarity, constant momentum flux, and a constant eddy viscosity. While the similarity assumption has extensive experimental support and is not subject to much question even in the more modern literature, some other assumptions have been and remain the subject of active research. Some of these will be discussed briefly here to indicate reasons that the computational results might not show perfect agreement with the classical jet theory, and in some cases why the computational codes might be expected to provide a better solution.

Intermittency. The shape of the turbulent jet can be roughly described as three regions, a central region, an extreme outer region, and an intermediate region. The central region is the fully turbulent region (assuming a location far enough along the axis that the initial development region has been passed) and encompasses about half the radial dimension between the axis of the jet and the edge. The extreme outer region is not part of 
the jet, but rather the ambient fluid, which in the present analysis and much of the literature is either described or set up experimentally as an irrotational flow region. The region between these two is what might be considered the mixing region. It has been studied extensively by researchers such as Townsend $13,14,15$ and provides the driving forces leading to entrainment of the ambient fluid into the jet region. The general nature of the mixing region is described by vortices that are generated along the edge of the jet which tend to entrain ambient fluid as they are stretched, expanded, and dissipated. Part of the nature of this region is that the boundary of the turbulent region is at any given time sharp and well defined, but its location changes rapidly with time. Therefore, a measuring device placed in this region is at times exposed to the turbulent flow of the central region and at times exposed to the irrotational ambient flow, the length of time for each being a function of the distance of the probe from the axis of the jet. The fraction of time spent in turbulent flow is a characteristic of the mixing region. It is referred to as "intermittency" and its study is well documented in the literature. 7,13,14, 22 The effect of intermittency on degrading the usefulness of the entrainment relationship developed above was recognized by Hinze, but he still felt the general behavior described by the entrainment relationship was qualitatively correct.

The work by Townsend described above is more detailed than the description in Hinze's book, ${ }^{7}$ but it describes physics of the edges of the jet that probably cannot be captured by the computational codes when the turbulence models are applied, and certainly are not captured by the classical theory against which the calculations are compared. Nonetheless, they provide better insight into the nature of turbulent flow and will be useful in trying to extend the basic analysis being described and validated here into the more complex situations required to analyze mixing in the HLW tanks.

Momentum flux / outer region flow.

The assumption of constant momentum flux in the turbulent jet was based on an assumption of no interactive forces between the jet and the ambient fluid. Since entrainment from the ambient fluid to the jet was observed experimentally, this required the flow from the irrotational region to the turbulent region to be perpendicular to the jet axis, so that no axial momentum was introduced into the jet as a result of the entrainment. This assumption has come under serious question by several researchers and it is probably not strictly true. Nonetheless, it is a usable concept that still provides a qualitative point of comparison for the calculations, but which at the same time shows the superiority of the computational results over the simple results obtained from the classical jet theory.

Kotsovinos ${ }^{10}$ published some of the earliest work questioning the accuracy of the constant momentum theory. He discussed the inadequacy of the constant momentum flux assumption in terms of the ambient irrotational flow and showed that the flow pattern induced by the entrainment of irrotational fluid into the jet was such that the inflowing fluid did in fact carry a component of momentum that reduced the total momentum in the jet. Simply put, this means that the jet had to apply a net force against the ambient irrotational flow region to entrain the fluid. Such a concept makes perfect sense on physical grounds, and corrections to 
the theory to account for this interaction served to explain discrepancies between measurements and the classical jet theory. Kotsovinos finds that a plane turbulent jet can lose as much as twenty percent of its momentum at a distance of 100 diameters from the jet nozzle. It is significant, though, that his work focuses on plane jets. Other accounts have indicated that while the reduction in momentum discussed by Kotsovinos is essentially correct, $16,17,18$ the details of the flow leading to that reduction, especially the interaction with the bounding walls of the irrotational region, are not accurate. Schneider ${ }^{17}$ indicates that the impact of the interaction between the turbulent jet and the irrotational flow is not as clear for the round jet as for the plane jet, that the effect is probably much smaller, and that there are few data to characterize this effect better for the round jet. Our interpretation of this field of study is that the momentum reduction makes good physical sense, but that the classical theory still provides a reasonable point of comparison, especially when one considers the uncertainties associated with the calculations. At the same time, the flow patterns shown by Kotsovinos, Schneider, and Zauner will prove valuable for qualitative comparisons with computational results.

$\boldsymbol{k}$ - $\varepsilon$ and other models . While the classical jet theory model described earlier is convenient for comparing with computational results, it is not a modern theory and does not provide the most up-to-date numerical approach to turbulence. Perhaps the most widely used numerical model is the $k-\varepsilon$ model, in which a turbulent kinetic energy and a dissipation rate of that energy are treated as transported properties and solved along with the conservation equations. A turbulent eddy viscosity is calculated from a combination of $k$ and $\varepsilon$ and is adjusted by a number of empirically determined constants. This viscosity is then used in conservation equations in the same manner as the turbulent eddy viscosity was employed in the classical theory.

Both FLUENT and CFDS-FLOW3D use the $k$ - $\varepsilon$ model as their fundamental turbulent flow model, so it is necessary to understand its usefulness and limitations. Both codes employ special $k$ - $\varepsilon$ models to accommodate calculations under those conditions when the basic model is known to be weak, such as flows near a bounding wall or flows with high curvature. More details of the $k-\varepsilon$ models will be given later.

An interesting application of the intermittency concept is that described by Cho \& Chung. ${ }^{19}$ They describe a model in which the intermittency, $\gamma$, has been incorporated into the $k$ - $\varepsilon$ model to determine an improved estimate of the eddy viscosity. They attempt to address two weaknesses of the conventional $k-\varepsilon$ model associated with the plane mixing layer but also related to the calculated behavior of the round jet. These include a computed mean velocity profile by the $k-\varepsilon$ model that approaches the free stream velocity too fast near the higher velocity region of the plane mixing layer and predicted Reynolds stresses in this higher velocity region that are too low compared to experimental measurements. In addition, they point to the "round jet / plane jet anomaly" in which the spreading rate is affected by the constants used in the model, and in which adjusting the constants for one jet configuration results in degrading the calculations for the other. 
The model developed by Cho and Chung employs an expression for intermittency based on a continuity equation for the turbulent fluid alone. It includes terms representing the spatial transport of intermittency owing to the mean velocity jump between the turbulent and irrotational fluids, generation of intermittency owing to the production of turbulent kinetic energy, transport of intermittency by spatial inhomogeneity or the gradient of the intermittency itself, and by transport of intermittency from the entrainment of mass from the irrotational flow into the turbulent fluid. The results of the intermittency equation were incorporated into the dissipation equation, which the authors identified as the principal source of the discrepancies between the $k$ - $\varepsilon$ model predictions and experiments. They discuss the effects of the change in terms of its effect on the calculated length scale of the turbulence, which subsequently has an impact of the calculated eddy viscosity.

Cho and Chung applied their model to a plane jet, a round jet, a plane far wake, and a plane mixing layer and showed improvement in the calculations for all of these shear flows compared to earlier models. While the intermittency modifications to the $k$ - $\varepsilon$ model are not available as options in either of the CFD codes used in the present work, the changes help point out potential weaknesses in the models employed here, and will serve to identify sensitivities that must be addressed in applying our results to tank mixing applications.

2.4 Relationship to $\boldsymbol{k}-\varepsilon$ models Excellent discussions of the development of increasing complex turbulence models and their relationships to one another are given by Arpaci and Larsen ${ }^{20}$ and Rodi. ${ }^{21}$ Rodi, ${ }^{21}$ Tennekes and Lumley, ${ }^{22}$ and Lumley ${ }^{23}$ discuss the limitations of the eddy viscosity models in general and the $k$ - $\varepsilon$ model in particular. The limitations notwithstanding, the $k-\varepsilon$ model enjoys the most widespread use of the turbulence models because of its somewhat more general nature than the classical mixing length models and its simpler formulation when compared to Reynolds stress computational models. The simpler nature of the $k-\varepsilon$ model translates directly into lesser requirements for computer resources, making it more convenient to use.

The mixing length theories of Prandtl and Boussinesq belong to the same family of "first order" turbulence models as the $k-\varepsilon$ transport equation model used in the codes. Their similarity is related to their approach to the momentum equation in that both models represent the Reynolds stresses in terms of an eddy viscosity, as first suggested by Boussinesq. The differences in the models stems from their approach to finding a value for the eddy viscosity. The classical models, at least as they have been described above, determine a constant value for the viscosity, based either on the geometry of the flow or on the characteristics of the jet behavior as determined by length and velocity scales. The $k-\varepsilon$ model is similar in that it uses a viscosity, but the determination is based on the solution of transport equations for both the kinetic energy of the flow and the dissipation of the kinetic energy. A dimensional analysis is then applied to combine these two transport parameters to infer an eddy viscosity. As might be expected, the model requires empirically determined constants which are dependent on the flow geometry. The $k-\varepsilon$ model differs from the classical models 
in that the value of the eddy viscosity changes with the flow conditions, whereas the classical models typically employ a constant eddy viscosity, albeit one determined from flow and geometry for a given application.

In their review of turbulence models, Arpaci and Larsen ${ }^{20}$ comment, “...all two-equation models remain part of the same family of models having rather similar characteristics and limitations." These limitations include calculations when the flow is near a wall or flows that turn from turbulent to laminar. The methods work best for parallel or nearly parallel flows at high Reynolds numbers. Therefore, they would be expected to give reasonable results for the application being discussed here, the turbulent free jet. Since the models being compared are from the same family of models as defined by Arpaci and Larsen, we expect that the results determined from the classical models and those calculated by the CFD codes are going to be similar. The adequacy of the methods, especially the $k-\varepsilon$ method when extended to the more complex geometries and flow conditions typical of mixing in the HLW tanks is yet to be determined. In addition, the extension to non Newtonian flows will have to be addressed in future reports, though one might note that the behavior of the $k-\varepsilon$ model is non Newtonian ${ }^{22}$ in that the eddy viscosity changes with the solution of the $k$ and $\varepsilon$ transport equations.

The $k-\varepsilon$ model is based on the conservation equations as shown in Equations (2.2) and (2.3). A mechanical energy equation is developed from the momentum equation and cast in the form, 24

$$
\frac{\partial}{\partial t}(\rho k)+\frac{\partial}{\partial x_{i}}\left(\rho u_{i} k\right)=\frac{\partial}{\partial x_{i}}\left(\frac{\mu_{t}}{\sigma_{k}} \frac{\partial k}{\partial x_{i}}\right)+G_{k}+G_{b}-\rho \varepsilon
$$

where $k$ is the turbulent kinetic energy, defined as

$$
k=\frac{1}{2} \sum_{i} \overline{u_{i}^{2}}
$$

A similar conservation equation for the rate of kinetic energy dissipation, $\varepsilon$, is

$$
\frac{\partial}{\partial t}(\dot{\rho} \varepsilon)+\frac{\partial}{\partial x_{i}}\left(\rho u_{i} \varepsilon\right)=\frac{\partial}{\partial x_{i}}\left(\frac{\mu_{t}}{\sigma_{\varepsilon}} \frac{\partial \varepsilon}{\partial x_{i}}\right)+C_{1 \varepsilon} \frac{\varepsilon}{k}\left(G_{k}+\left(1-C_{3 \varepsilon}\right) G_{b}\right)-C_{2 \varepsilon} \rho \frac{\varepsilon^{2}}{k}
$$

where $C_{1 \varepsilon}$ and $C_{2 \varepsilon}$ are empirical constants, $\sigma_{k}$ and $\sigma_{\varepsilon}$ are "Prandtl" numbers governing the turbulent diffusion of $k$ and $\varepsilon, G_{k}$ is the rate of production of turbulent kinetic energy

$$
G_{k}=\mu_{t}\left(\frac{\partial u_{j}}{\partial x_{i}}+\frac{\partial u_{i}}{\partial x_{j}}\right) \frac{\partial u_{i}}{\partial x_{j}}
$$

and $G_{b}$ is the generation of turbulence due to buoyancy 


$$
G_{b}=-g_{i} \frac{\mu_{t}}{\rho \sigma_{h}} \frac{\partial \rho}{\partial x_{i}}
$$

The eddy viscosity arises from the velocity and length scales inferred from the solution to the $k$ and $\varepsilon$ transport equations. The velocity scale is taken as $\sqrt{k}$, while the length scale is $\sqrt{k^{3}} / \varepsilon$. These two scales are combined in the general form of Eq. (2.14), which shows the eddy viscosity as having the dimensions of a length scale times a velocity scale. Multiplying the two scales determined from the $k$ and $\varepsilon$ equations gives

$$
\mu_{t}=\rho C_{\mu} \frac{k^{2}}{\varepsilon}
$$

It is interesting to note that this value of the turbulent eddy viscosity is not constant, but rather changes with the solution of the conservation equations. This makes the flow behavior as represented by the $k-\varepsilon$ model non Newtonian. ${ }^{7}$

\subsection{Reference data for this report. Data against which to benchmark the} results of the present calculation will be taken from Kiser. ${ }^{25}$ They represent experimental measurements designed to measure the structure of the mean flow and the mean concentration field. Only the mean flow data are of interest in this report.

The experiment run by Kiser was a 3/8-inch submerged jet of a dilute solution of sodium chloride driven by gravity from a supply tank into a quiescent large volume of only slightly less salt concentration. The water jet entered the tank with a velocity of $3.66 \mathrm{~m} / \mathrm{sec}$ and a Reynolds number based on the jet diameter of about 35,000. The experiment is described in enough detail in Reference 25 to allow a computational model to be constructed.

The data provided by Kiser include measurements of the axial distribution of total head, velocity, and concentration as a function of axial distance from the jet injection point. $\mathrm{He}$ also includes measurements of the half-widths of the mean velocity and concentration profiles. Measurements of the radial distribution of the mean velocity are provided, and these will be compared against the similarity profiles determined from the calculations as well as from the classical jet theory. Kiser goes on to provide plots of the eddy viscosity coefficient for both the velocity and concentration profiles, but his determination is tied directly to the turbulence model he used to interpret the data. Since his eddy viscosity coefficient results are not data, but rather the results of calculations, they will not be used for direct comparisons in this report.

Other experimental work (as referenced in $\mathrm{Hinze}^{7}$ ) show a velocity profile obeying a Gaussian distribution, $\bar{u}_{z} / \bar{u}_{z, \max }=\exp \left(-108 \xi_{2}^{2}\right)$. The observation of a Gaussian profile is common when describing the radial distribution of axial velocity, and many researchers have 
fit their results with such a curve. This appears to be more a curve fitting convenience than anything else, but it is so commonplace that a comparison will be shown in this report for completeness.

Further data from Hinze associated with the behavior of a gas jet in air show

$$
\begin{aligned}
\bar{u}_{z} & =0.017 \bar{u}_{z, \max } & & \text { at } \xi_{2}=0.06 \\
& =0 & & \text { at } \xi_{2}=0.127
\end{aligned}
$$

and a spreading angle of

$$
1 / 2 \alpha=7.24^{\circ} \text {. }
$$

While these data are associated with a turbulent jet in air, there is much evidence that air and water jets behave similarly. ${ }^{26}$ Therefore, these air data can used for yet another point of comparison.

\section{Computational Code Analytical Models}

\subsection{Conservation equations.}

The fluid conservation equations used in the codes are the same as those described above. They are not cast in exactly the same form, though, so they will be written here in the form used in the FLUENT User's Guide. ${ }^{24}$

\section{Conservation of mass:}

$$
\frac{\partial \rho}{\partial t}+\frac{\partial}{\partial x_{i}}\left(\rho u_{i}\right)=S_{m}
$$

\section{Conservation of momentum:}

$$
\frac{\partial}{\partial t}\left(\rho u_{i}\right)+\frac{\partial}{\partial x_{j}}\left(\rho u_{i} u_{j}\right)=-\frac{\partial p}{\partial x_{i}}+\frac{\partial \tau_{i j}}{\partial x_{j}}+\rho g_{i}+F_{i}
$$

where the stress tensor, $\tau_{i j}$, is given as

$$
\tau_{i j}=\left[\mu\left(\frac{\partial u_{i}}{\partial x_{j}}+\frac{\partial u_{j}}{\partial x_{i}}\right)\right]-\frac{2}{3} \mu \frac{\partial u_{l}}{\partial x_{l}} \delta_{i j}
$$

Even though the current analysis is isothermal, the energy equation must be included to represent the turbulent energy transport mechanisms. 


\section{Conservation of energy}

FLUENT solves the conservation of energy equation in terms of conservation of the static enthalpy, $h$, defined as

$$
h=\sum_{i^{\prime}} m_{i^{\prime}} h_{i^{\prime}}
$$

where

$$
h_{i^{\prime}}=\int_{T_{r e f}}^{T} c_{p, i^{\prime}} d T
$$

$T_{\text {ref }}$ is a reference temperature and $c_{p, i}$ is the specific heat at constant pressure for species $i^{\prime}$.

Under an assumption that species diffusion due to pressure and external forces is negligible, the energy equation becomes

$$
\frac{\partial}{\partial t}(\rho h)+\frac{\partial}{\partial x_{i}}\left(\rho u_{i} h\right)=\frac{\partial}{\partial x_{i}}\left(k \frac{\partial T}{\partial x_{i}}\right)-\frac{\partial}{\partial x_{i}} \sum_{j^{\prime}} h_{j^{\prime}} J_{j^{\prime}}+\frac{\partial p}{\partial t}+u_{i} \frac{\partial p}{\partial x_{i}}+\tau_{i j} \frac{\partial u_{i}}{\partial x_{j}}+S_{h}
$$

$T$ is the temperature, $\tau_{i j}$ the viscous stress tensor, $J_{j}$ the flux of species $j^{\prime}$, and $k$ the mixture thermal conductivity. $S_{h}$ is an enthalpy source term.

Turbulent transport equations are found from the conservation equations above using a Reynolds averaging process. Using an overscore to indicate a time averaged value and expressing fluctuating values with a prime, a general conservation equation for the turbulent transport of a scalar $\phi$ is,

$$
\frac{\partial}{\partial t}(\overline{\rho \phi})+\frac{\partial}{\partial x_{i}}\left(\overline{\rho u_{i} \phi}\right)=-\frac{\partial}{\partial x_{i}}\left(\bar{\rho} \overline{u_{i}^{\prime} \phi^{\prime}}\right)+\overline{D_{\phi}}+\overline{S_{\phi}}
$$

Note that a diffusion term, $D$, has been included in this general transport equation. The averaging process has assumed that the necessary integration time interval is longer than the largest time scale of the turbulent fluctuations and that the fluctuations in the scalar property, $\phi^{\prime}$, are random, so that the time average of those fluctuations is zero.

The $k-\varepsilon$ turbulence model includes two more transport equations, one for $k$ and one for $\varepsilon$, as described earlier. The discussion in Section 2.4 was taken from the FLUENT manual, so it will not be repeated here. 


\section{CFDS-FLOW3D:}

The conservation equations in CFDS-FLOW $3 D^{27}$ are quite similar to those in FLUENT, as might be expected. The continuity equations are the same with the exception of a source term included in the FLUENT equation that is not shown in the CFDS-FLOW3D manual. This is probably not a significant difference because mass sources can be added to this equation through the use of user specified subroutines.

The momentum equations are the same and require no further comment. The energy equations are somewhat different. The energy equation as written in the CFDS-FLOW3D manual is

$$
\frac{\partial \rho H}{\partial t}+\nabla \cdot(\rho U H)-\nabla(\lambda \nabla T)=\frac{\partial p}{\partial t}
$$

where $H$ is defined by

$$
H=h+\frac{1}{2} U^{2}
$$

The variable $H$ is described as a total enthalpy, and as such both FLUENT and CFDSFLOW3D cast the energy equation in terms of enthalpy. However, the enthalpy $H$ as defined by CFDS-FLOW3D is not a standard definition. It adds the kinetic energy, $(I / 2) U^{2}$, to the thermodynamic enthalpy, $h=h(T, p)$, whereas the FLUENT formulation uses just the thermodynamic enthalpy, $h$. This results in the different appearance of the FLUENT and CFDS-FLOW3D energy equations, Equations (2.20) and (2.21), even though the equations are essentially identical. There are two reasons for the apparent differences. One is the fact that CFDS-FLOW3D includes the kinetic energy as part of its "enthalpy." While this definition of total enthalpy is not standard, the assumptions are clearly stated and physically acceptable. The second reason for apparent differences is simply the way the authors of the code manuals decided to present the equation. FLUENT includes all the terms that can be used in the energy equation through input options, even though conduction, mass diffusion, viscous stress, and source terms are not included in the default energy equation. On the other hand, CFDS-FLOW3D shows the default form of the energy equation. All of the terms available in the FLUENT formulation are also available in the CFDS-FLOW3D formulation, again as input options. Therefore, the energy equations in the two codes are the same.

The transport equations representing the $k-\varepsilon$ model in the two codes are virtually identical. The differencing schemes and the options available for different types of differencing are also similar between the codes. The calculations described in this report utilized similar differencing options in all cases. 


\subsection{Significant physical observables}

While the CFD codes being used in this analysis have good output capabilities; there are limitations to what parameters can be observed. Some of the key variables of interest can be plotted with relative ease. These include: the decay of axial velocity with distance along the jet axis; the radial distribution of axial velocity with distance along the jet axis, or the similarity profiles; the spreading angle of the jet as measured by some defined criterion to be specified during the analysis; and the entrainment rate, which is also affected by the determination of the edge of the jet. All of these comparisons are expected to be reasonably good, both between the individual calculations with the two computational codes and between the codes and the classical turbulent jet model described above.

Further comparisons with variables representative of the turbulent jet behavior include plots of both $k$ and $\varepsilon$. Both codes will allow these to be plotted, but neither code is expected to show a particularly good comparison with the classical turbulent jet model. The principal reason for this is the assumed constant eddy viscosity in the classical model, as compared to the varying eddy viscosity in the $k-\varepsilon$ model used by the codes. The comparisons will be used to help interpret the results and draw conclusions about the applicability of the codes when extended to applications beyond the turbulent free jet.

While the codes require an input of turbulence intensity and a length scale, these values are not available as standard output. Because of the nature of the $k-\varepsilon$ model, the fluctuating component of the velocity is not computed, so the turbulence intensity is not available, except as inferred through the turbulence kinetic energy. No attempt will be made to interpret turbulence intensity results in this report, although a comparison of turbulent kinetic energy with data will be made. Similarly, shear stress profiles are not available as standard output, except perhaps at the walls of the region. These results are not of interest in the current study, so they will not be addressed in the analysis.

\subsection{Options in the codes}

Both codes have similar options for turbulence models, the most basic of which is the $k-\varepsilon$ model discussed here. Other more sophisticated models included in both codes are the Reynolds stress model and a more modern version of the $k-\varepsilon$ model based on renormalization group theory, the RNG $k-\varepsilon$ model. Both of these models have the potential of giving better results for particular applications. In the present case, the turbulent free jet, the standard $k-\varepsilon$ model is expected to work as well as the other two, but in future analyses involving the influence of bounding walls, rotating jets, or the transition from turbulent to laminar flow, the other models may show significantly better performance. In particular, the RNG $k-\varepsilon$ model will be studied in some detail, since it is supposed to provide a significant improvement over the standard $k$ - $\varepsilon$ model at only a modest increase in computer running time. The Reynolds stress models are supposed to require significantly more computer resources, thereby making them less attractive. In addition, the Reynolds stress models supposedly require more 
empirical parameters to be input that does the RNG $k-\varepsilon$ model. In the current environment of limited physical data to describe the materials in the HLW mixing tanks, this makes the RNG $k$ - $\varepsilon$ model more attractive. Detailed comparisons among the various turbulence models have not been made for this report, although some effects of using the RNG $k-\varepsilon$ model in FLUENT are noted.

\section{Computational models}

\subsection{Basic grid setup}

The basic working models for both the FLUENT and CFDS-FLOW3D analyses used approximately 20,000 nodes. The FLUENT model was set up as a 1/4-section of the experimental rig, with 25 active nodes in each coordinate direction perpendicular to the jet axis, the $x$ - and $y$-directions, and 30 active nodes along the jet axis, the $z$-direction. Since FLUENT adds a row of "dead" cells at each boundary, this resulted in a $26 \times 26 \times 31$ cell model, or 20956 cells. The nozzle region of the jet was modeled with four cells spanning a distance of $4.7625 \mathrm{~mm}$ ( $3 / 16$ inch), the nozzle radius. The remaining 21 cells each direction perpendicular to the jet axis spanned the remainder of the $0.3048 \mathrm{~m}$ of the experimental facility. Thirty cells were used in the axial direction to span $1.2 \mathrm{~m}$, the length of the experimental facility. Figure 2 shows a drawing of the surface cells for this model, while Figure 3 shows an expanded view of that portion of the inlet plane representing the jet and the immediately adjacent cells. While the grid is a structured grid, the cell sizes are not uniform. They are most densely packed in all three coordinate directions in the vicinity of the incoming jet, and they spread out in each direction as the jet spreads. The spreading rate is gradual to avoid excessive differences in the dimensions of adjacent cells, and the rate of spreading in each coordinate direction is similar so as to maintain acceptable aspect ratios in each cell. The entire grid was checked by the FLUENT internal checking algorithm for acceptable skew and aspect ratios for all cells in the grid. While some cells were identified with excessive skew, there was only a small number and the grid was accepted.

The CFDS-FLOW3D computational model was similar in concept to the FLUENT model, but the method used to distribute the mesh cells was different. A drawing analogous to Figure 3 is shown for the CFDS-FLOW3D model in Figure 4. It can be seen that the CFDS-FLOW3D grid structure conforms more closely to the initial round shape of the incoming jet, but the difference between this and the more rectangular shape of the FLUENT model grid did not seem to have a significant impact on the calculated results. The CFDS-FLOW3D model used five cells in the nozzle region, while the FLUENT model used four. It is possible that this caused some of the differences seen later in the initial behavior of the jet, but a more significant difference was the use of body-fitted coordinates (BFC) in the CFDS-FLOW3D to expand the mesh as the distance perpendicular to the jet axis increased.

Both the FLUENT and CFDS-FLOW3D grids were developed using the BFC option to capture the circular shape of the jet nozzle, and both grids were expanded to cover the 


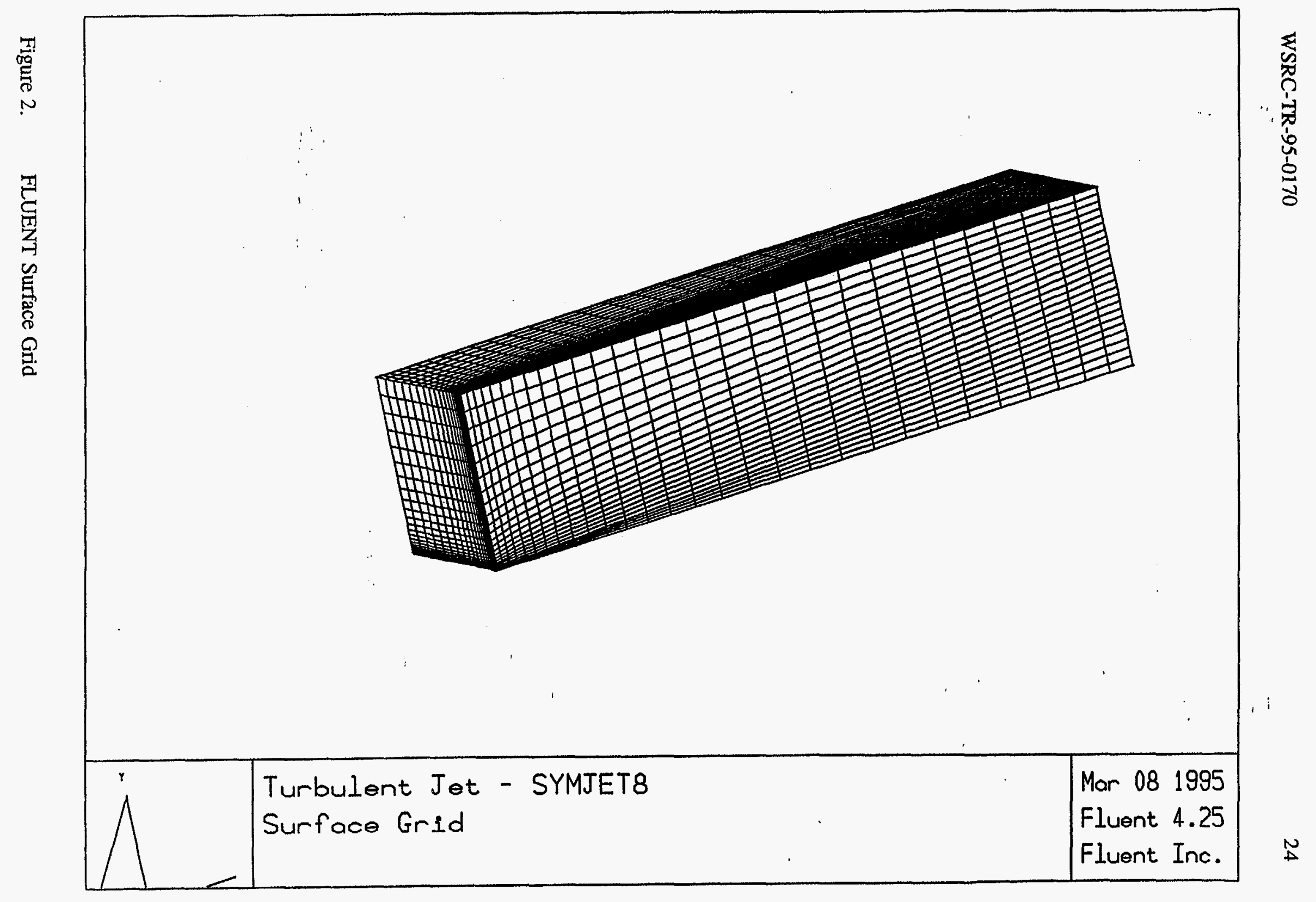




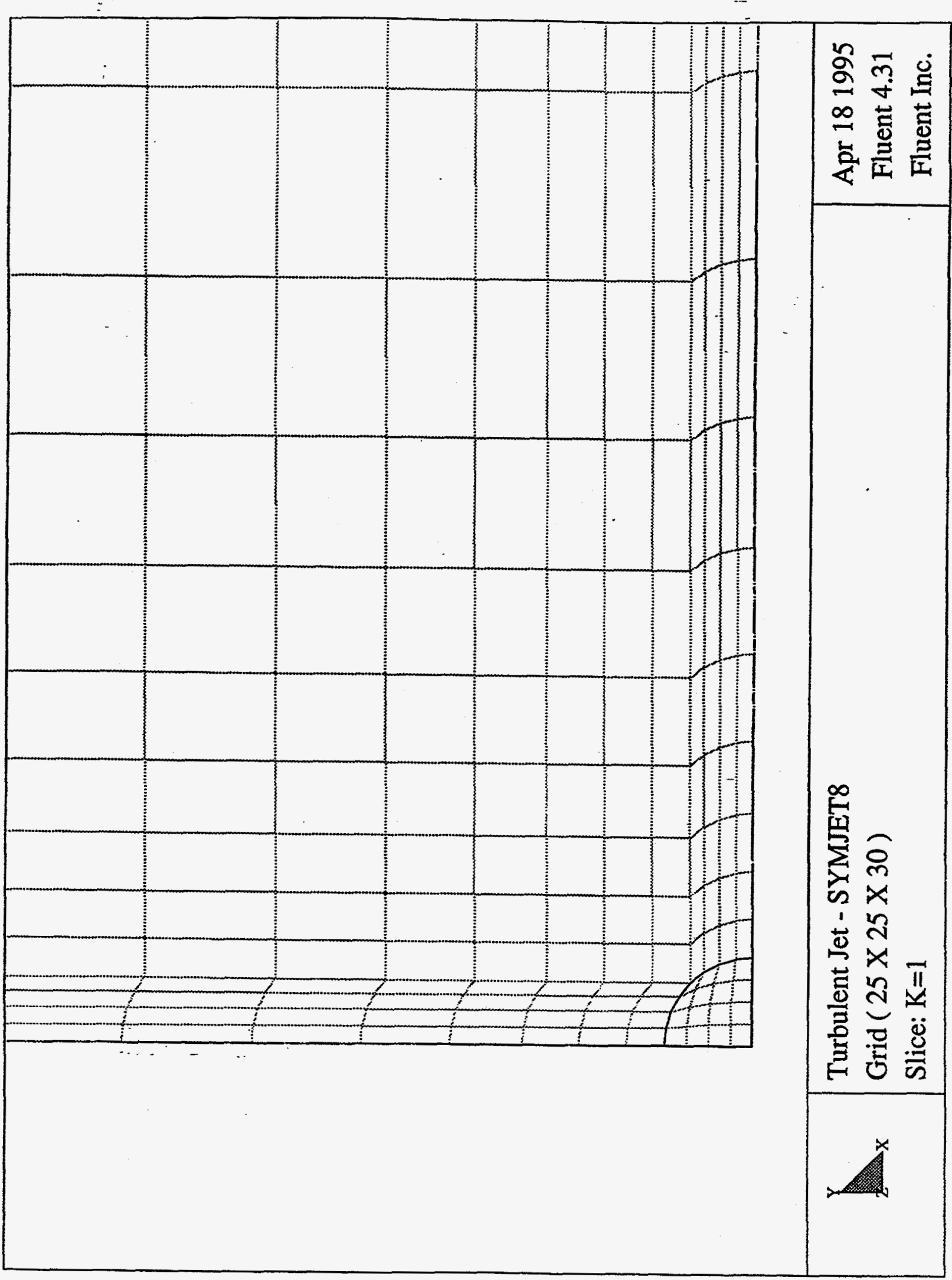

Figure 3. FLUENT Inlet Plane. Grid snapshot near the jet nozzle. 


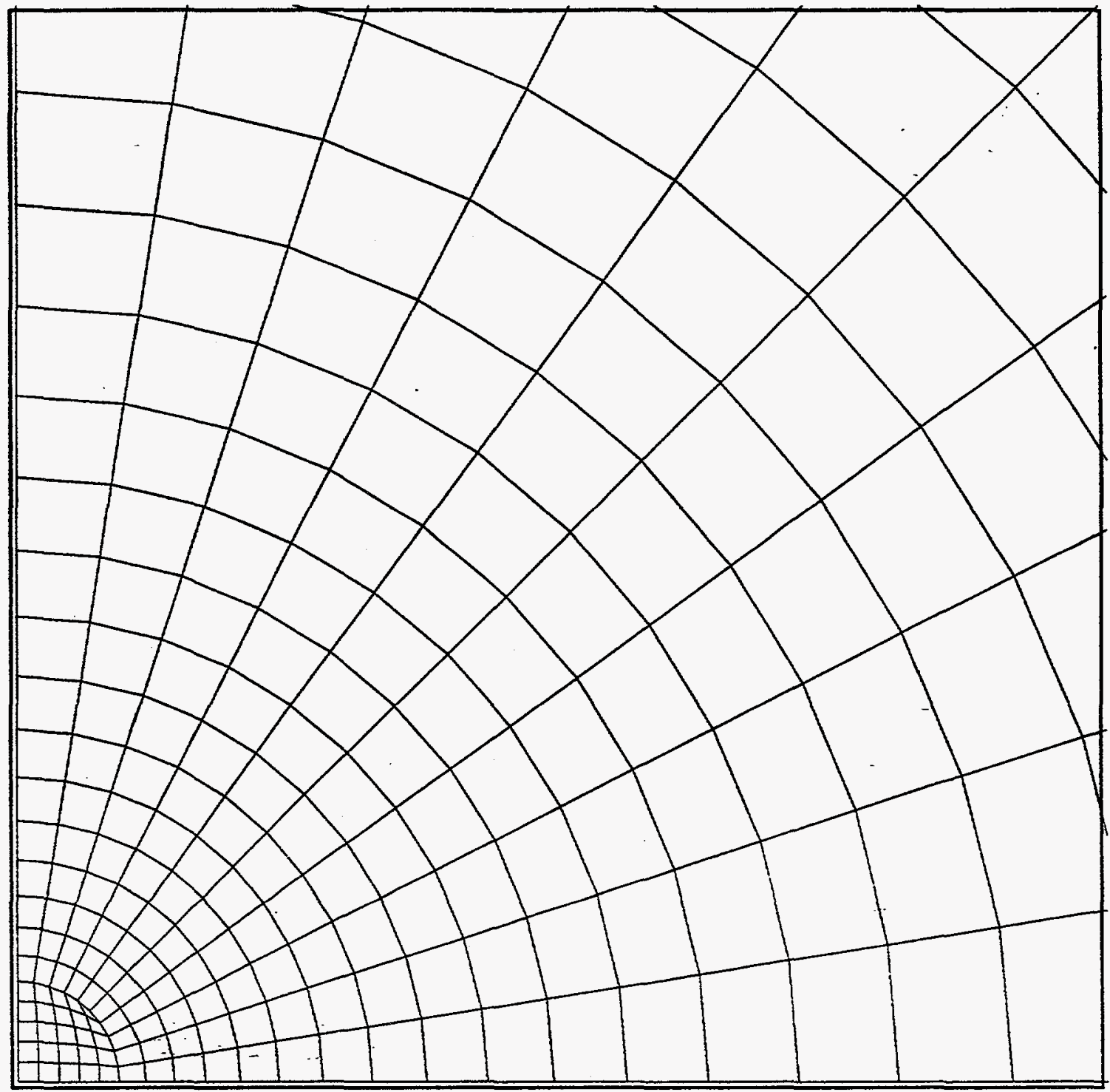

Figure 4. CFDS-FLOW3D Inlet Plane. Grid snapshot near the jet nozzle. 


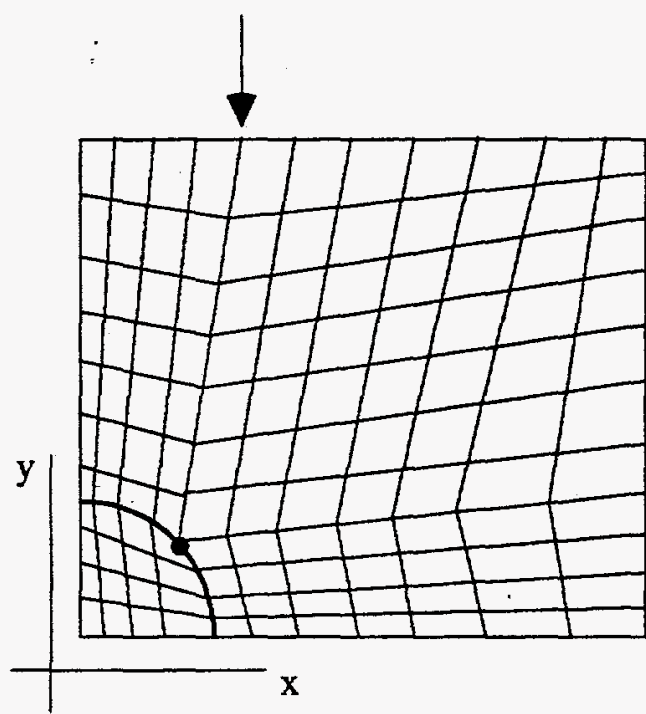

FLUENT

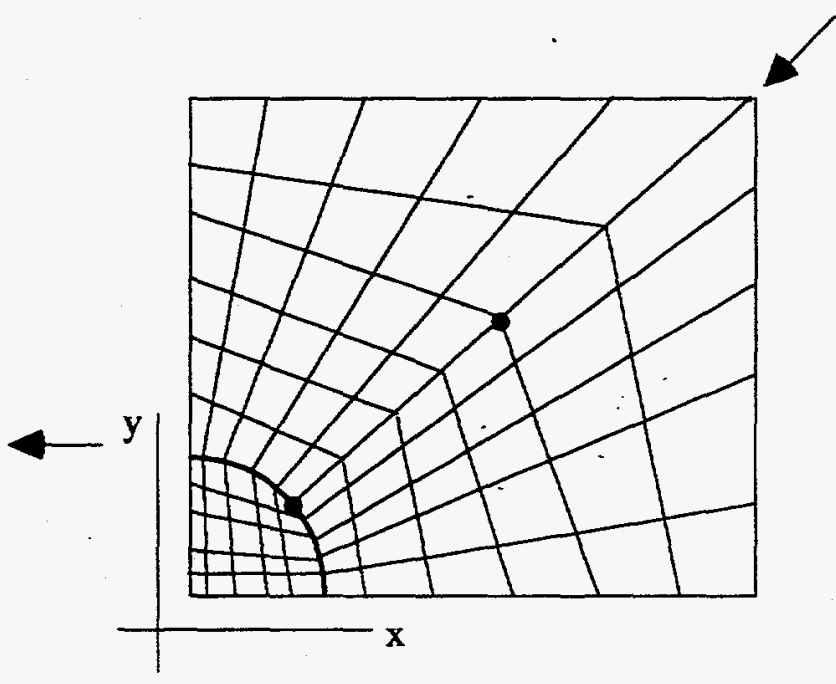

CFDS-FLOW3D

Figure 5. Grid expansion schemes

rectangular shape of the experimental facility. However, the method used to expand the grids was different. The FLUENT model used the same number of grid points on the $x$ and $y$ axes bordering jet as it did on the on the opposite edges of the computational domain, as shown in the sketch of the grid structure in Figure 5. For example, 25 cells were used to define the $y=1$ edge of the inlet plane and 25 cells were used on the opposite edge of the inlet plane at $y=26$. The edges are the top and bottom edges in Figure 5. The actual grid pattern near the nozzle is shown in the partial drawing of the FLUENT inlet plane in Figure 3 while the expansion of the grid is illustrated in Figure 5. The edge of the nozzle is shown as the heavy circular line in the FLUENT drawing in Figure 5, greatly expanded here for clarity. The dot in the center of the arc represents a node used to break the circular arc into two segments, one associated with the $x$-dimension and the other with the $y$-dimension. The arrow at the right side of the diagram shows the expansion of the grid from the edge of the jet to the edge of the computational domain. The remaining nodes along the right side are redistributed to cover the entire edge, but the total number of nodes on the right edge can be seen to be the same as the number on the left edge. The same is true for the top and bottom edges.

The CFDS-FLOW3D grid is different. Again, the edge of the jet nozzle is shown as a heavy line in Figure 5. The nodalization in the interior region of the jet is similar to the FLUENT model, except five cells are used instead of four. Notice that in the CFDS-FLOW3D model, the five cells describing the edge of the jet are expanded to cover the entire span of the right side of the computational domain. This is different from the FLUENT model and results in a much coarser nodalization in that region of the model outside of the jet, namely the ambient region into which the jet expands. The CFDS-FLOW3D model used 39 cells to model the left and bottom edges as shown in Figure 5, while the right and top edges were modeled with only five cells. (Figure 5 is only a sketch and does not show the actual number of cells used in 
either model.) The CFDS-FLOW3D model used a total of 365 cells in each plane perpendicular to the jet axis, while the FLUENT model used 625.

Recall that the nominal overall dimension of both models was about the same, about 19,000 cells if only the live cells are counted. The difference in node density in the planes perpendicular to the jet axis means that the CFDS-FLOW3D model used a finer nodalization along the jet axis than the FLUENT model, 52 cells rather than 30 used in the FLUENT model. This difference is thought to have affected the development region of the jet flow in the calculations. Figure 6 shows the axial nodalization from the CFDS-FLOW3D model. The FLUENT model was similar.

\subsection{Parameters and boundary conditions}

To reduce the size of the computational grid and still maintain adequate detail, 1/4-symmetry of the experimental tank about the jet axis was assumed. This means that the corner of the nozzle region in Figure 2 represented the axis of the jet. With the computational $z$-axis extending along the jet axis, both the $x$ - and $y$-coordinate planes then represented planes of symmetry in the calculation. In the CFD calculations, this forced a zero velocity gradient at these planes. The tangential velocity along these symmetry planes was unrestricted. The exit plane was treated as a pressure boundary in both models. This specification sets a user specified ambient static pressure which is used as a bounding pressure in the calculations. Any velocity head calculated by the codes for an outflow condition at the exit plane is included in addition to the static boundary pressure, so the total (or stagnation) pressure at the outlet is different from the specified static boundary pressure.

The differences between the models was the treatment of the bounding walls parallel to the jet axis. The CFDS-FLOW3D calculation treated the side walls as "wall" boundaries. This specification imposes a zero normal velocity and a no-slip boundary condition at the walls. While this is an accurate representation of the experiment for the side walls, it is not accurate for the top wall, which in fact was open to the atmosphere in Kiser's ${ }^{25}$ experiment. However, it represents the bottom of the tank properly. The difficulty in representing the top and bottom of the experiment separately (and properly) arises from the symmetry boundary condition along the lower coordinate plane of the model. This forces the top and bottom boundaries in the calculation to be the same. This was considered to be an acceptable approximation given that the grid would have to be doubled to remove it.

The FLUENT analysis tested the sensitivity of the calculated results by imposing a different set of boundary conditions. The first case used pressure boundaries on all bounding surfaces. The pressure boundary is flexible in that flow is allowed to enter or leave the solution space through these boundaries. A second asymmetrical case was run in which the side walls were represented as wall boundaries in the same manner as the CFDS-FLOW3D 
WSRC-TR-95-0170

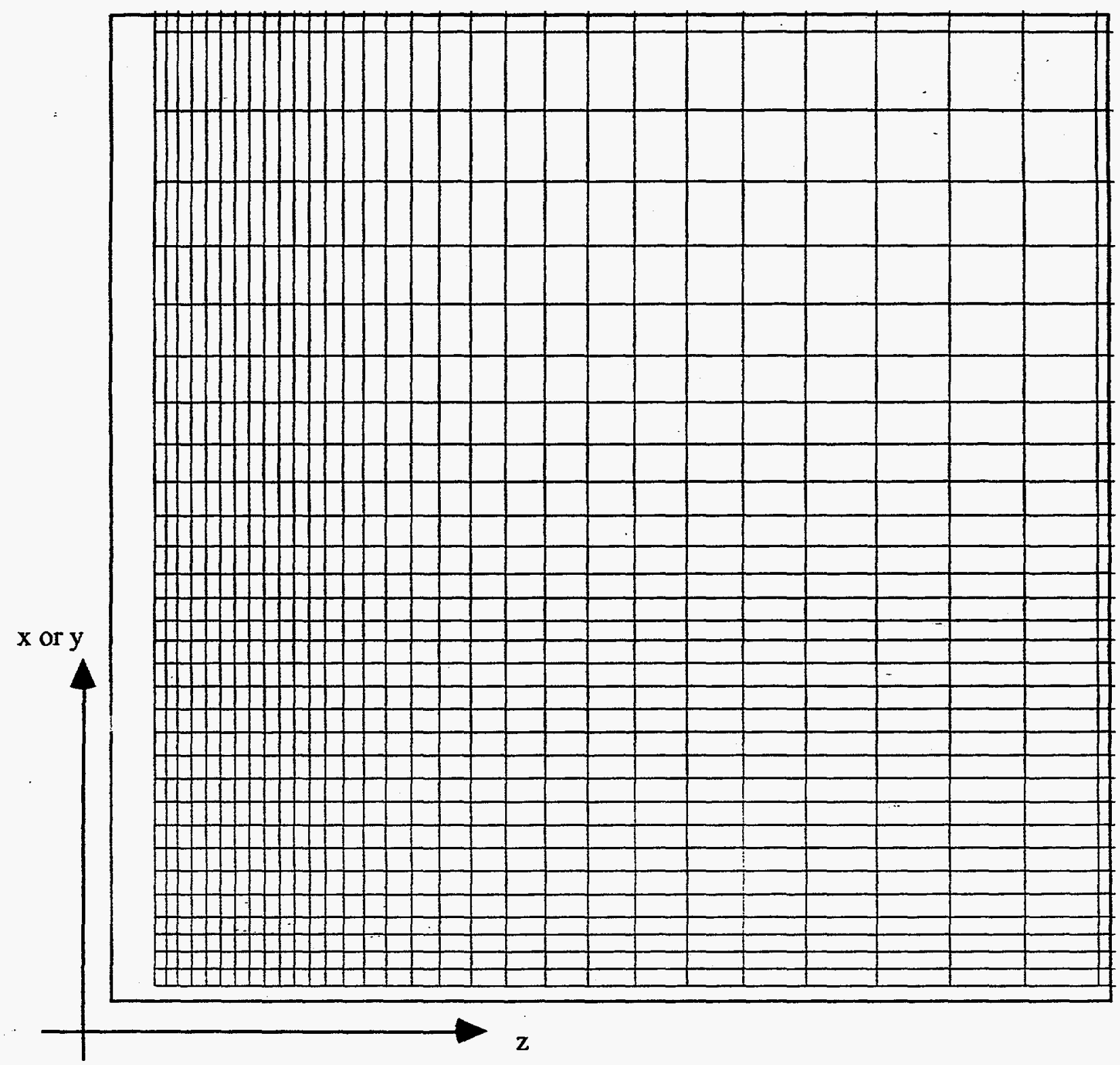

Figure 6. CFDS-FLOW3D axial grid structure. 


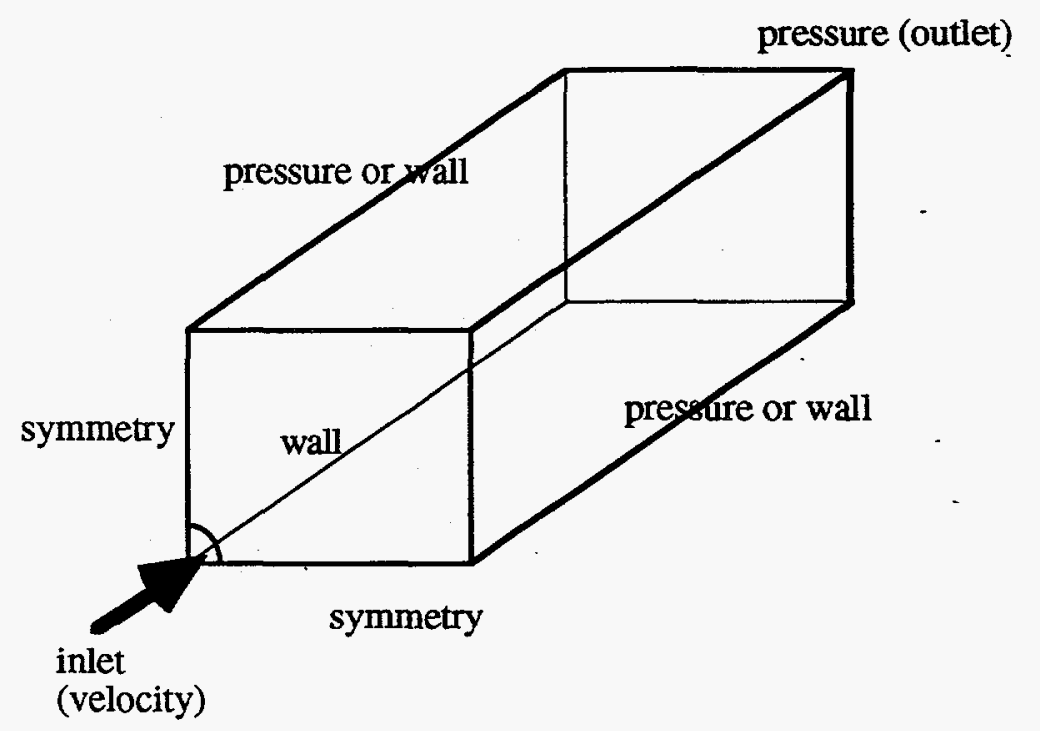

Figure 7. Boundary conditions for the 1/4-geometry model.

model, but the top (and therefore, the bottom) surfaces were represented as pressure boundaries. It will be seen later in the analysis that while the jet variables along the axis were not affected by the boundary condition specifications, the small flows defining the edge of the jet, and the flow patterns representing these flows, were significantly affected by the boundary conditions. Figure 7 shows the general arrangement of the boundary conditions for the codes.

\subsection{Sensitivity calculations}

Since computational results can be influenced by the mesh used to perform a calculation, it is necessary to ensure that results are physically correct and not an artifact of the grid scheme. Therefore, the FLUENT and CFDS-FLOW3D calculations were subjected to sensitivity runs in which the number of cells used to calculate the turbulent jet behavior was changed. The FLUENT calculations were repeated on a coarser mesh, one in which the number of nodes in each of the coordinate directions was approximately halved. The resulting model used about 2700 nodes.

The CFDS-FLOW3D model was modified in the other direction; i.e., more nodes were used to test the results on a finer mesh. The 20,000 cell model was modified to 60,000 nodes by reducing the dimensions of the cells in each of the coordinate directions by about $2 / 3$. It is interesting to note that for such a small change in the linear dimension of each of the nodes, the mesh size tripled. This results not only in greater computer memory requirements, but also significantly more run time needed to complete the analysis. This sensitivity calculation 
serves to emphasize the need to determine the coarsest mesh possible to address the phenomena of interest when the models developed here are extended to the global tank mixing analysis. The results of the mesh sensitivity calculations will be included with the analysis presented in the next section.

\section{Analysis of results}

\subsection{Qualitative results}

The general jet behavior predicted by the codes is just what was expected - a uniform spreading pattern, development of similarity profiles after the initial development region is past, and an entrainment flow along the edges of the jet. Figure 8 shows the development of the jet pattern in the surrounding fluid along the axis of the jet. The light colored quarterround section in the upper left hand corner of the figure is the inlet nozzle, the lighter colors in the figure representing higher velocities. A rough measure of the velocities is given by the gray scale shown at the left of the figure. The line extended from the nozzle downward to the right is the jet axis, so the figure shows the one-quarter of the jet that was calculated. The expansion of the light region along the axis is the calculated expansion of the jet, and the gradual dissipation of the very light region along the axis shows the dissipation of the axial velocity as the jet progresses into the surrounding fluid. The round nature of the pattern is obvious, even though these results are taken from the case with asymmetrical boundary conditions. Clearly, the boundaries are too distant to significantly impact this flow distribution. Figure 8 shows every other plane from $k=1$ to $k=13$ in the FLUENT calculation. This represents a distance of about $0.3 \mathrm{~m}$ along the jet axis, or about 30 nozzle diameters.

Radial velocity profiles along the jet axis are also as expected. Two locations are shown in Figures 9 and 10, the inlet plane and the exit plane for the FLUENT symmetrical boundary condition case. Note in both cases that the flow pattern within the turbulent region is outward, while the flow pattern outside the turbulent jet region is radially inward. The relatively quiescent region shown in Figure 10 corresponds closely to that radial position which gives a constant momentum flux carried within the jet region. It is important to recognize that the velocities shown in these two figures are small compared to the jet nozzle velocity. This can be seen by comparing the scales shown in Figures 8, 9, and 10. In particular, notice that the radial velocities shown at the exit plane, Figure 10 , are about three orders of magnitude smaller than the nozzle velocity. Therefore, while these plots show the nature of the flow distribution and the entrainment behavior of the jet, the impact of this behavior on mixing so far removed from the jet nozzle will be negligible. Note that the axial velocity has been omitted in these profile plots so that the velocities perpendicular to the axis can be distinguished.

The results for CFDS-FLOW3D are quite similar. A plot analogous to Figure 8 is shown in Figure 11, except the velocity vectors are shown along the jet axis. The radial distribution of 


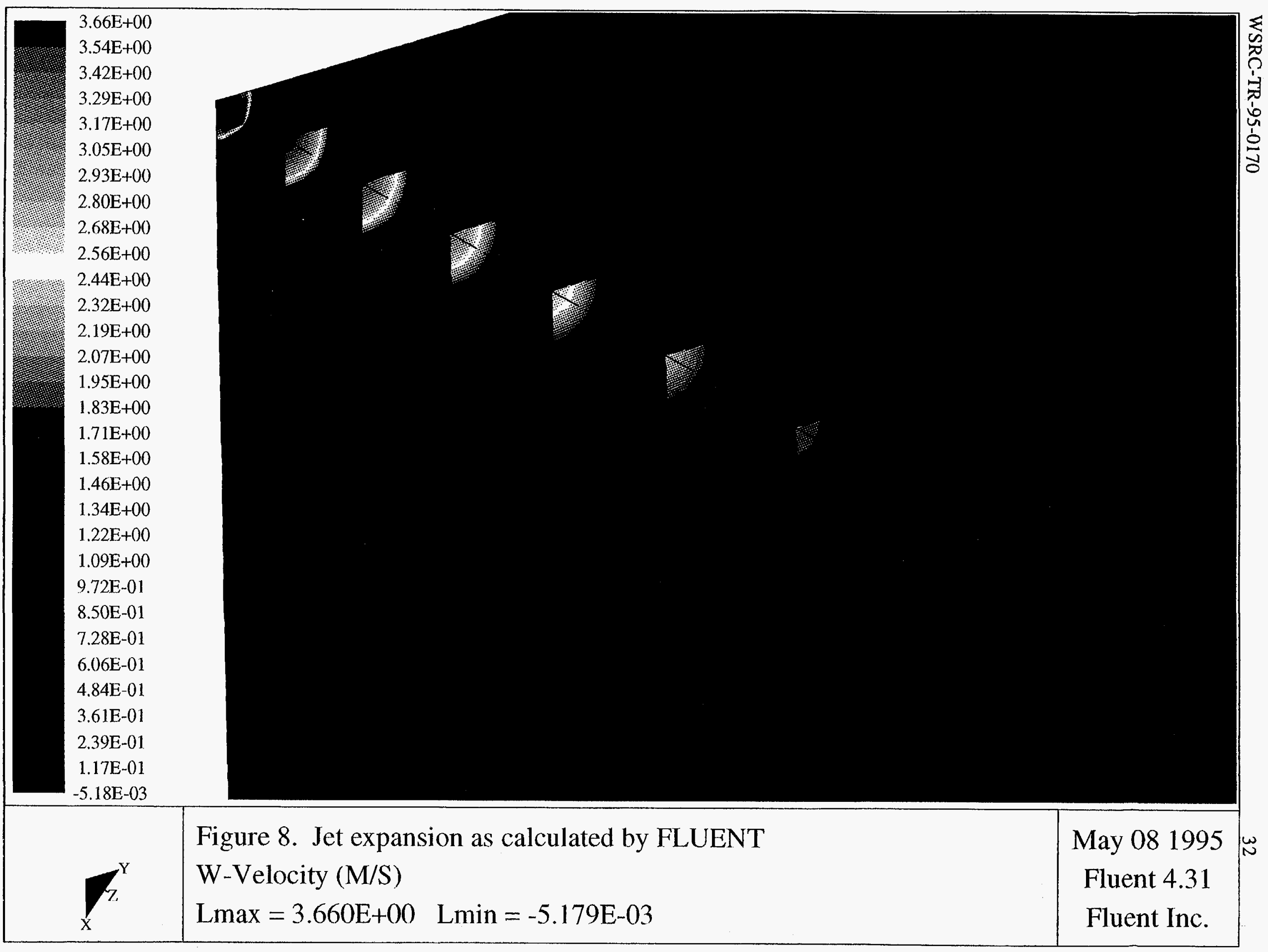




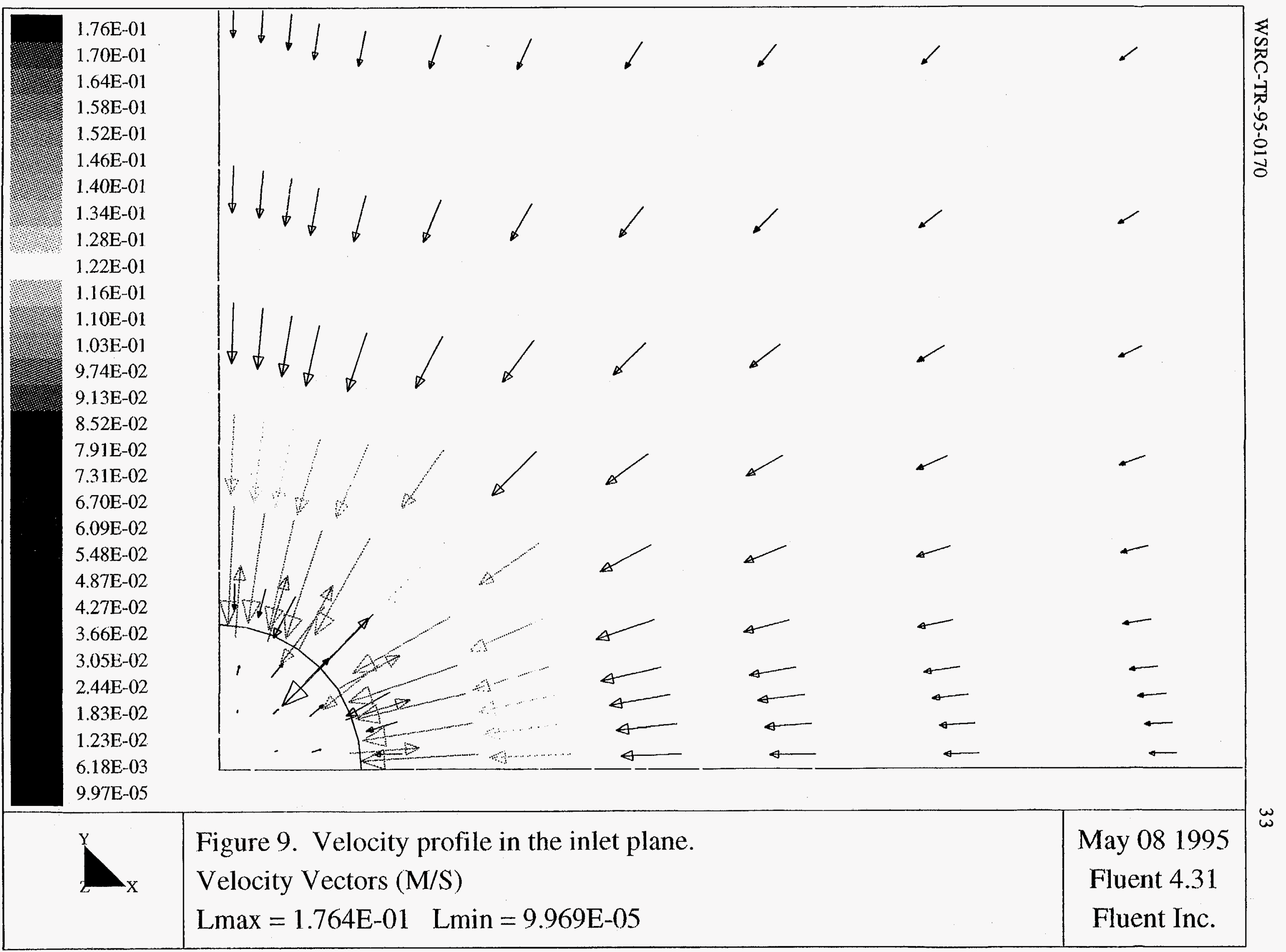




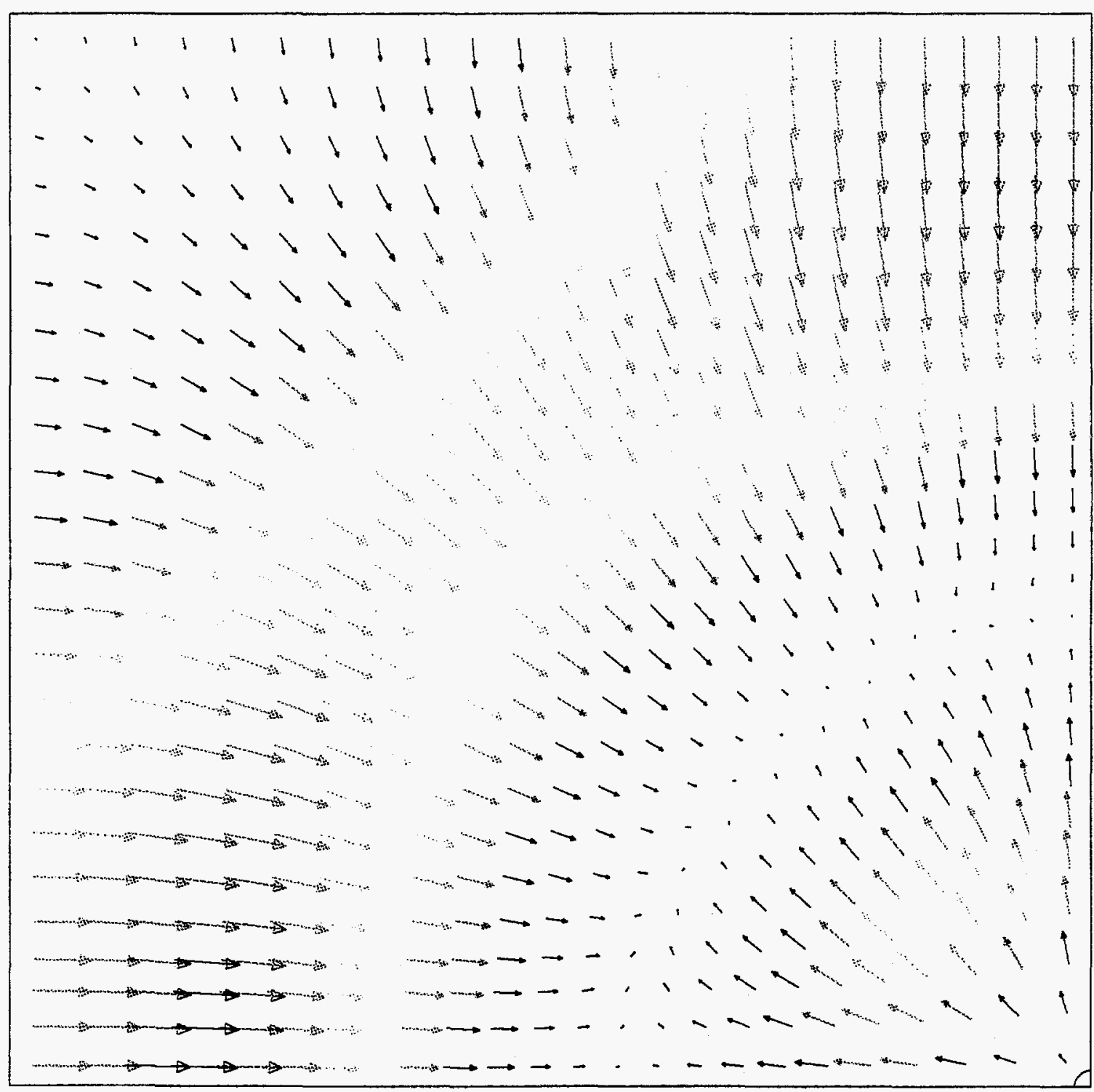

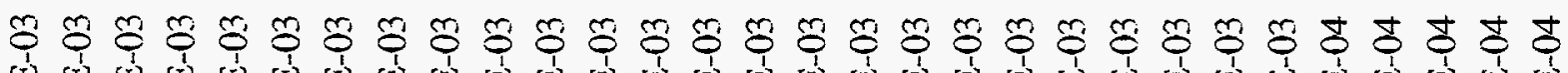

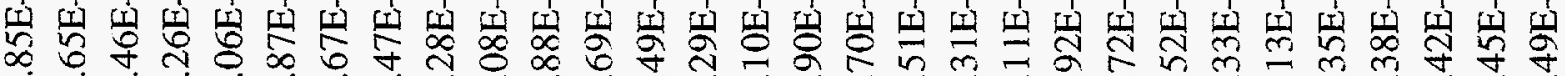

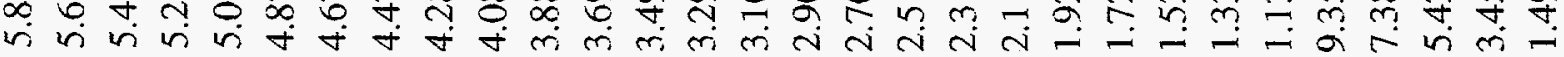


WSRC-TR-95-0170

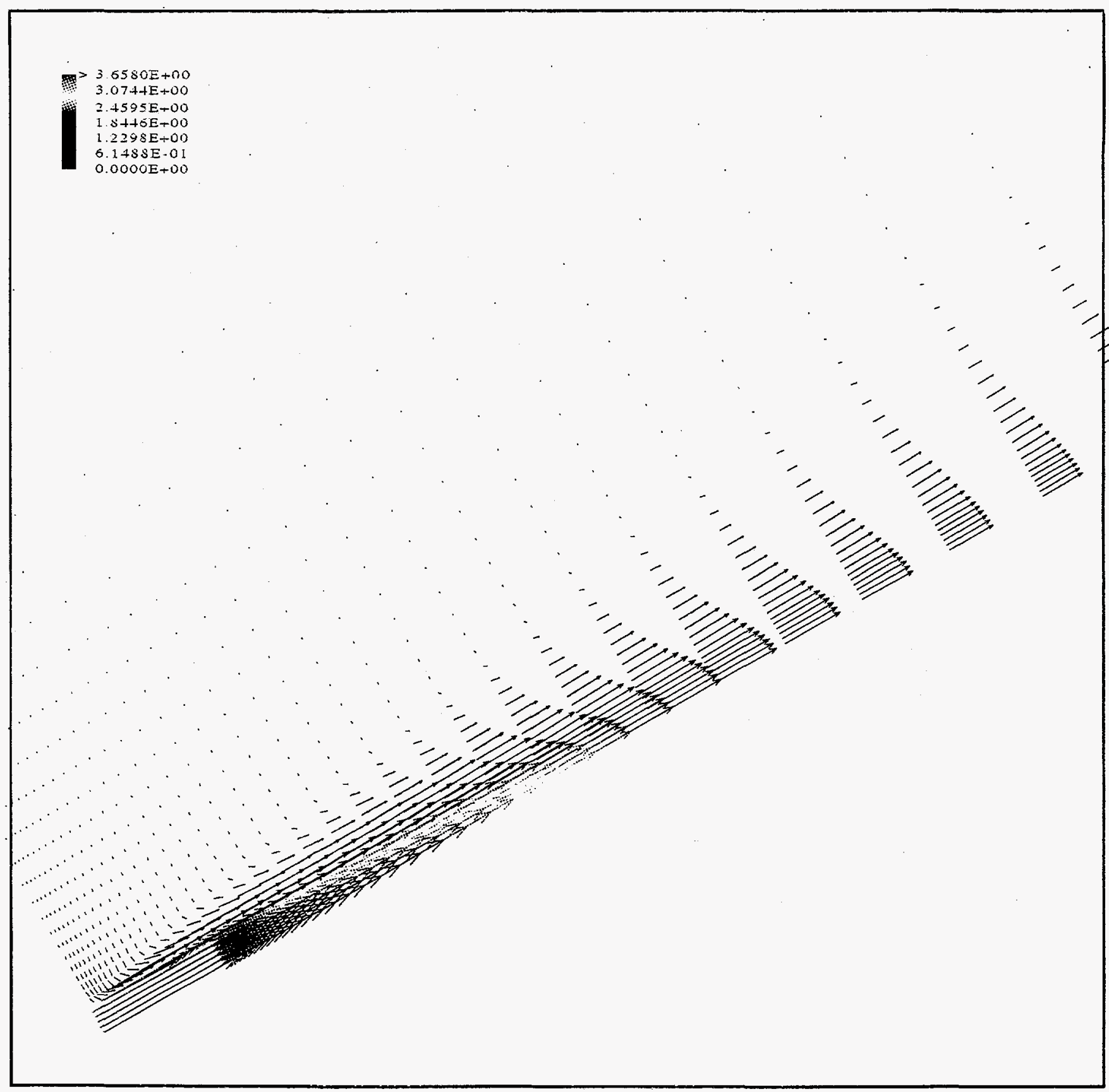

Figure 11. Jet expansion in the CFDS-FLOW3D calculation. 
axial velocity as the jet spreads is obvious. In addition, the small entrainment velocity along the edges of the jet can be observed. The quantitative nature of these profiles will be compared later in this section.

The velocity profile in planes perpendicular to the jet axis were harder to observe with CFDSFLOW3D because the axial velocity could not be omitted from the plot. In Figure 12, the inlet plane is shown, with the apparent crosshatch region representing flow into the page from the nozzle. The small radially inward velocities can be observed in the ambient fluid. Figure 13 represents a plane about halfway along the jet axis. The spreading effect can be seen, as well as the entrainment flow. Both of these results are in good qualitative agreement with our expectations from classical jet theory.

Figures 14 and 15 show the impact of the boundary conditions on the perpendicular velocity profiles. These figures are taken from the asymmetrical case in which the side walls were modeled as wall boundary conditions (no slip) and the top boundary condition was a pressure boundary. Figure 14 shows no distinguishable difference at the inlet plane (compare to Figure 9), but Figure 15 shows that the jet has been distorted toward the wall boundary at the exit plane (compare to Figure 10). While it is clear that the boundary conditions affect the jet profile, the radial velocities are small compared to the axial velocity along the jet axis (or even at the jet periphery, for that matter) and the effect on mixing behavior is probably small. Therefore, we don't consider this observed asymmetry important for the tank mixing applications.

\subsection{Axial velocity behavior}

A much more important parameter for tank mixing applications is the dissipation of the jet velocity along its axis. The behavior of the codes is compared to data and classical models in Figure 16. It is apparent from this graph that the results of the codes, the classical models, and the data are all in reasonable agreement with each other, although there are several differences that warrant further investigation.

The first observation is that the CFDS-FLOW3D calculation shows an entry region over which there is essentially no spreading. In fact, the calculated velocity increases a bit over the first five or six diameters into the tank. FLUENT, on the other hand, begins to dissipate the flow immediately. There was a difference in the models at the entrance region in that the CFDSFLOW3D model included a very short (less that one nozzle diameter) blockage along the edge of the jet to ensure that the velocity was directed uniformly along the jet axis as is entered the tank. The FLUENT model, while specifying a uniform axial velocity as the inlet boundary condition, did not include this effective wall along the edge of the jet for the small distance into the tank. We do not know if that caused the difference in initial spreading. Another possible cause for the quicker spreading, and probably more likely, is that the FLUENT mesh grows more rapidly away from the nozzle region than does the CFDSFLOW3D model. The mesh density near the nozzle could affect the ability of the code to 


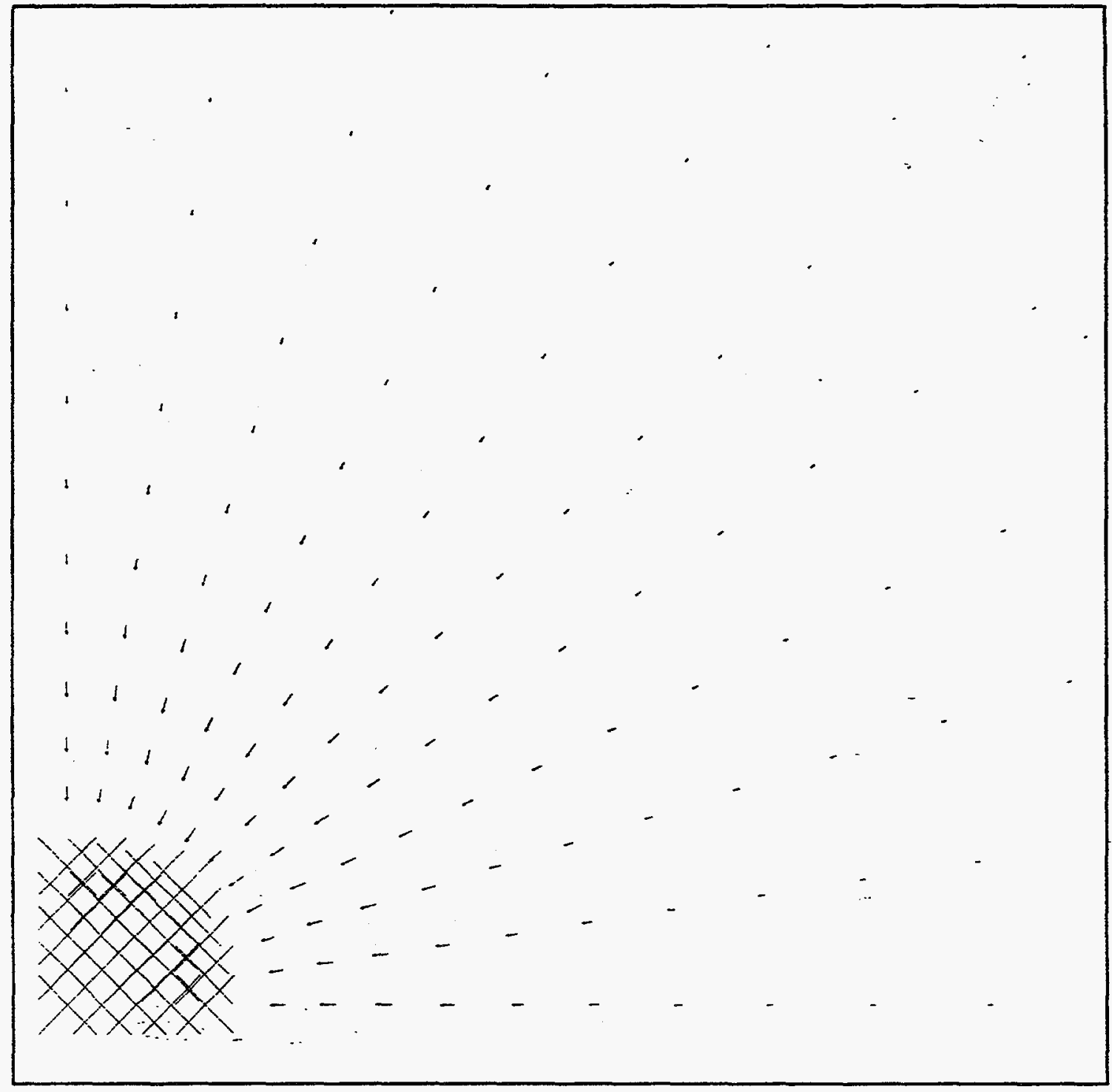

Figure $12 . \quad$ Velocity vectors in the CFDS-FLOW3D inlet plane. 


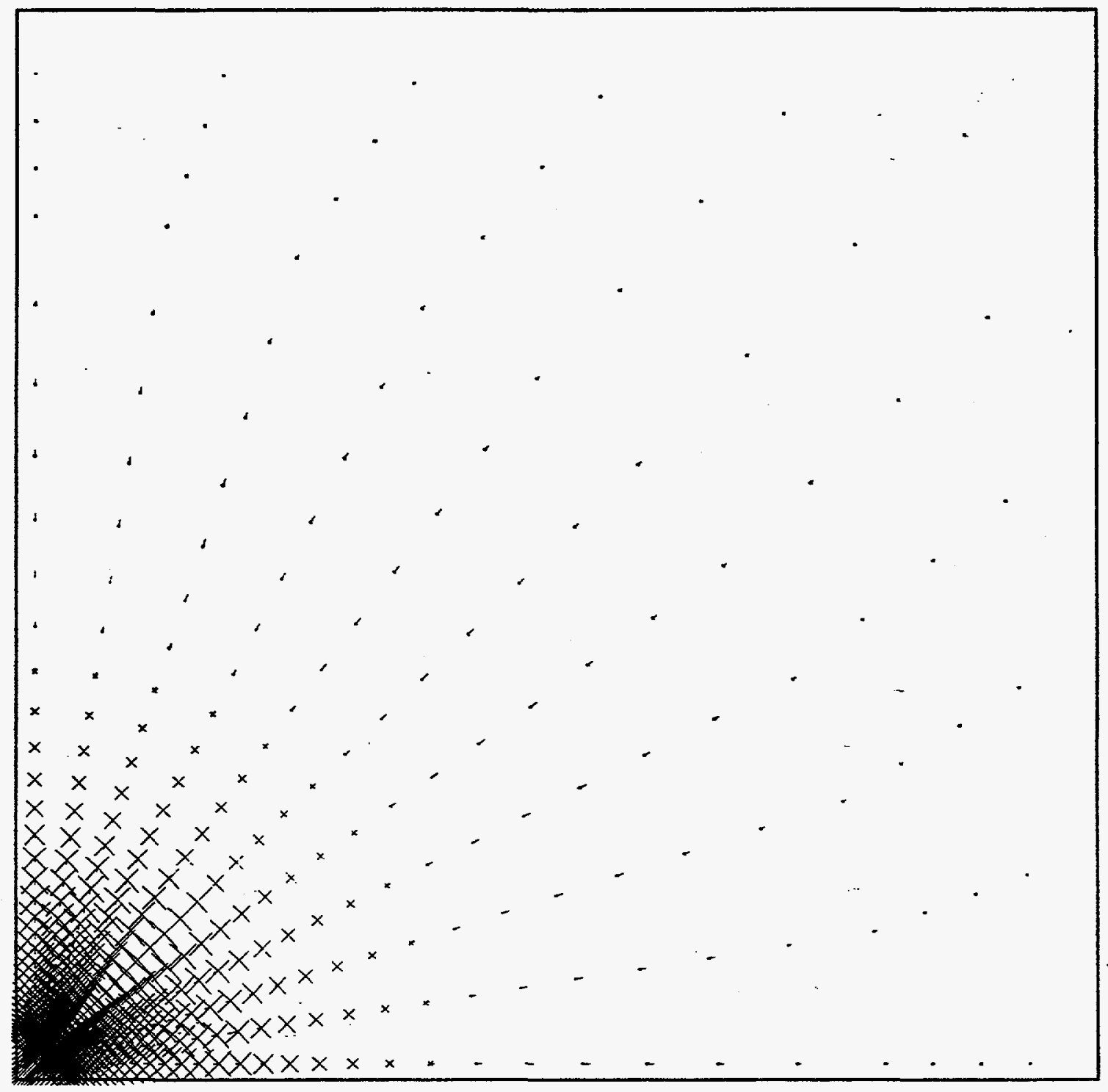

Figure $13 . \quad$ Velocity vectors in a CFDS-FLOW3D middle plane. 


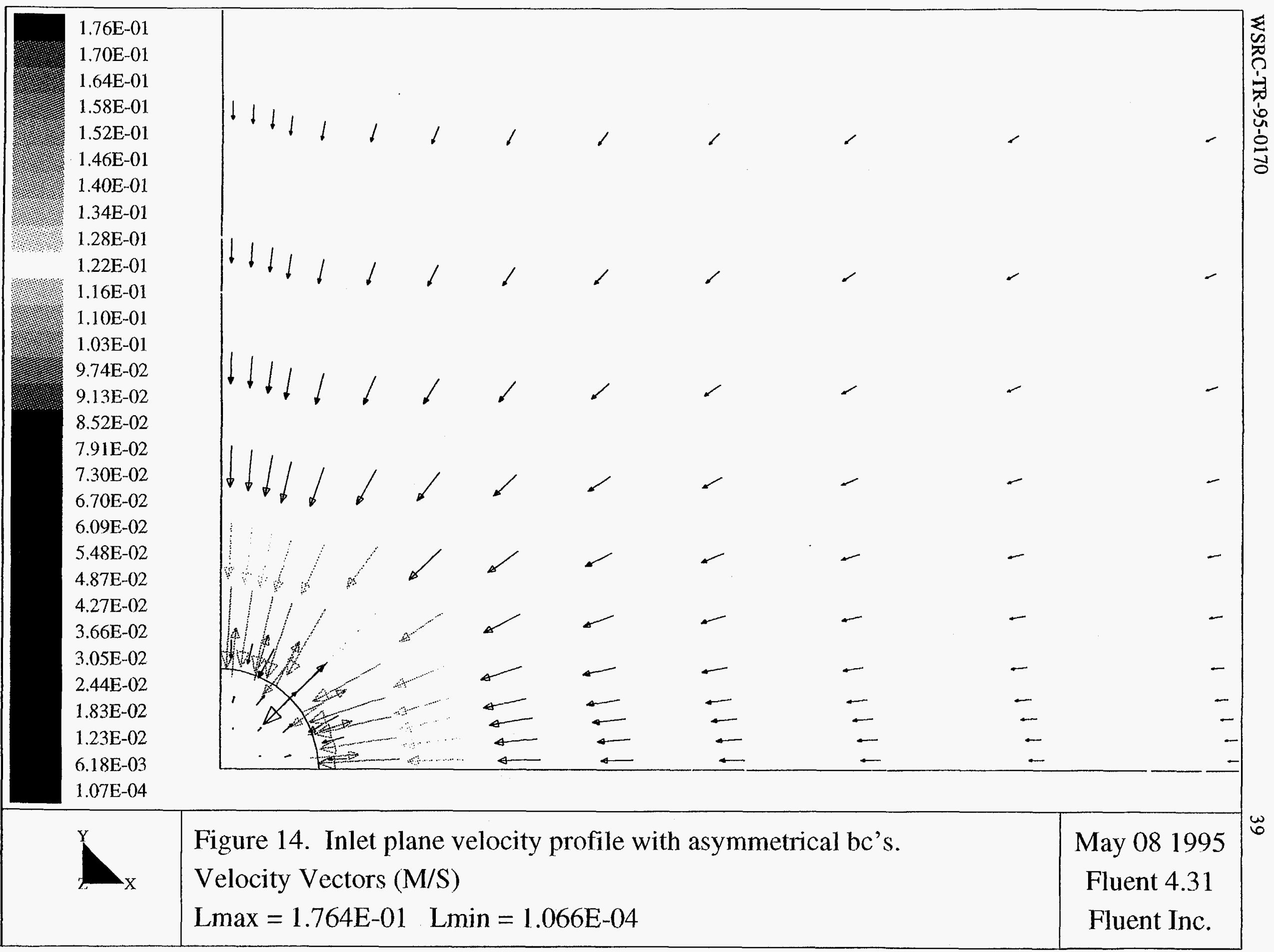




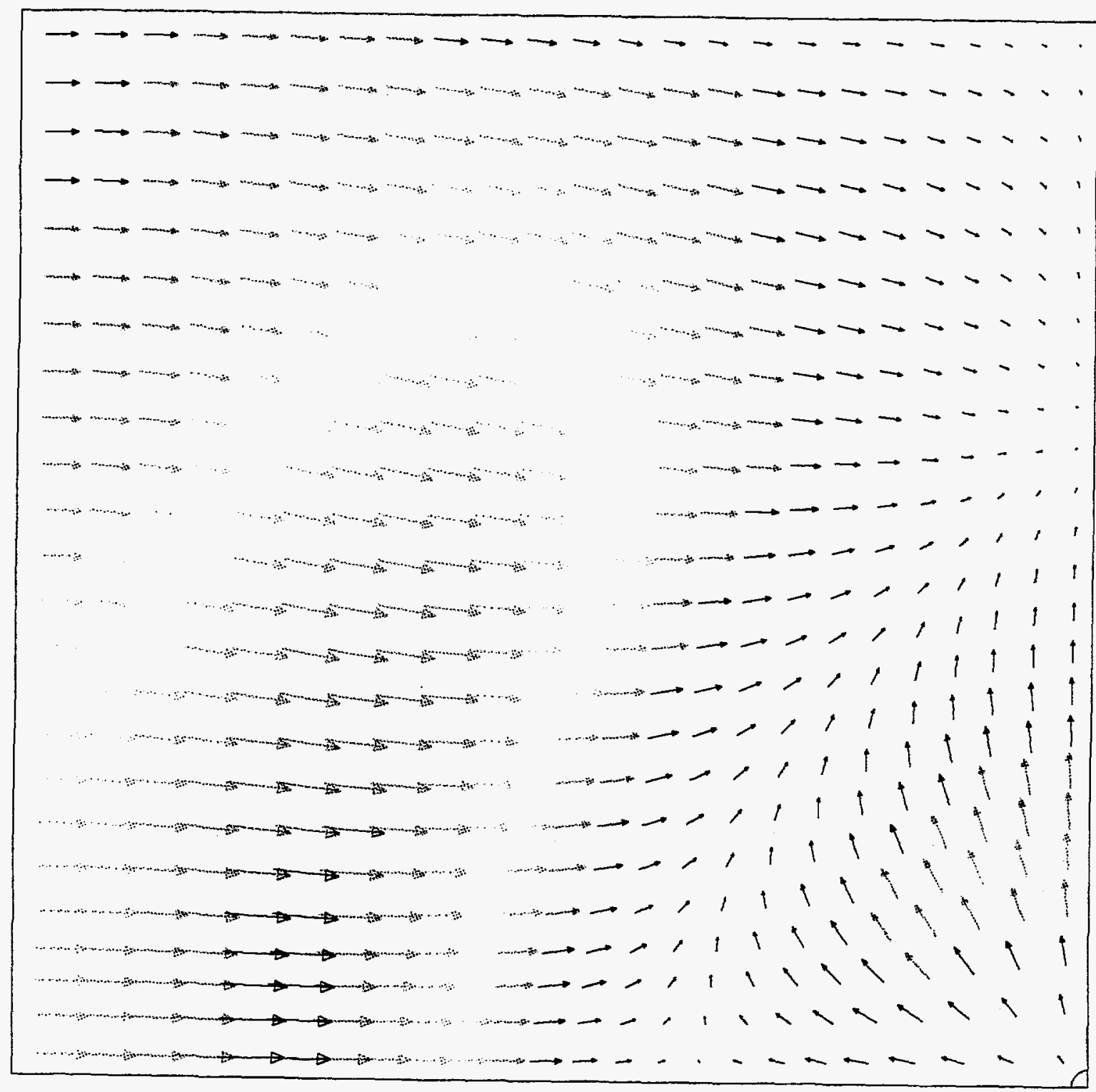

ชి

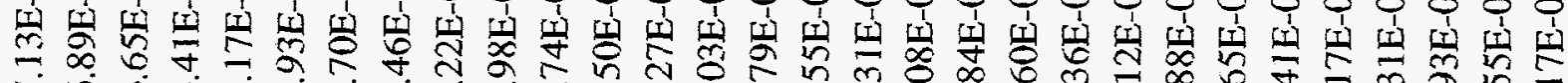

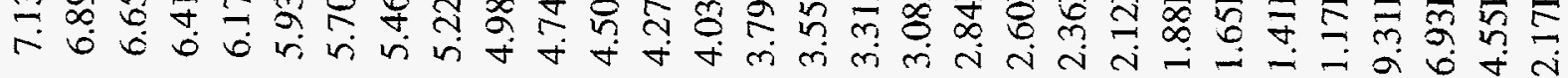




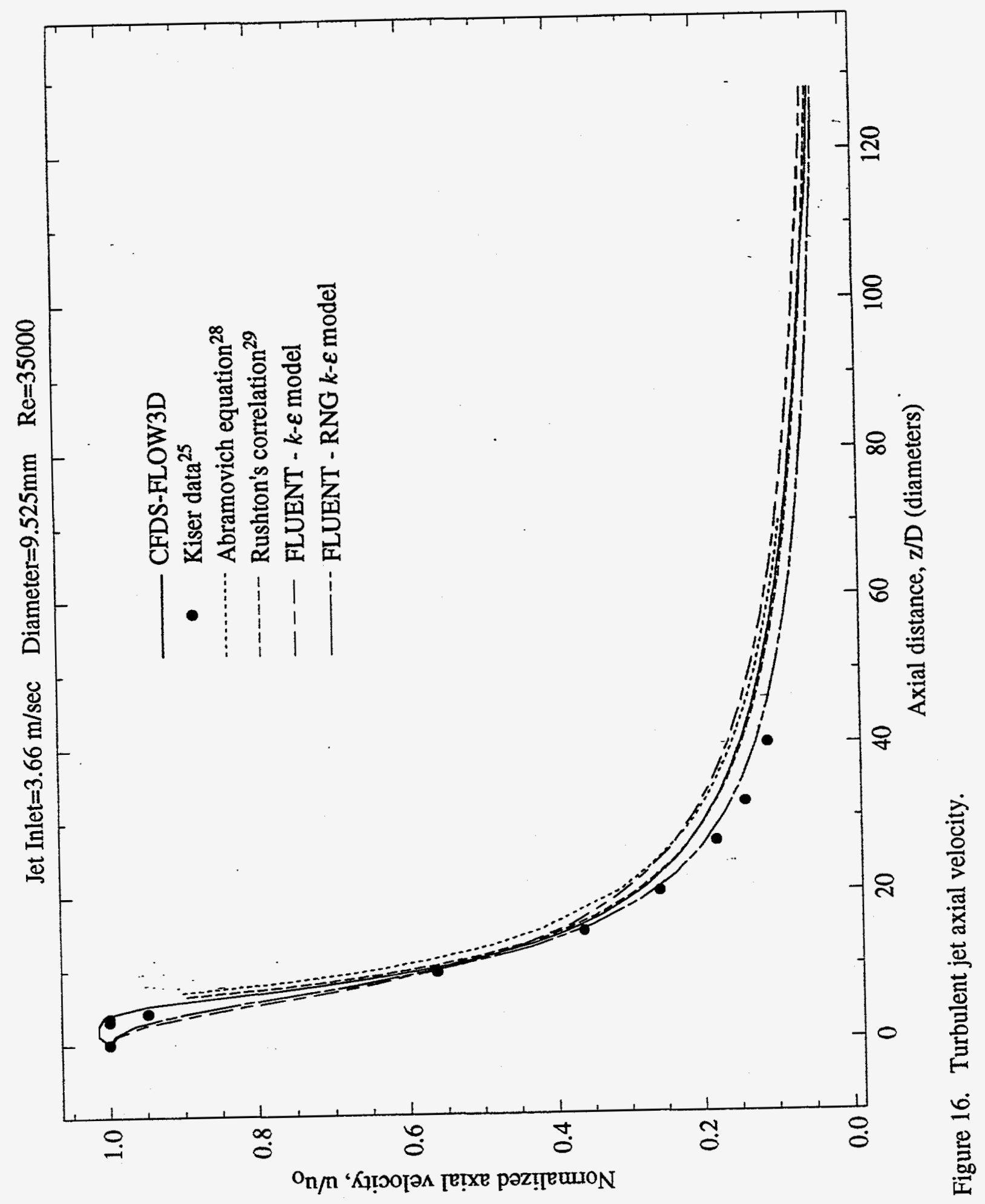


capture the boundary behavior between the jet and the ambient fluid as the jêt emerges from the nozzle. The effect of this mesh design will be investigated more as work continues with the wall jet problem later in this project.

Once the initial development region is past, the behavior of the two codes is quite similar. The axial velocity profile far from the jet nozzle is about the same for both codes, and both show higher velocities than the data in the range of $10-40$ diameters. While this is not our expected result, both codes are consistent. We expected the $k-\varepsilon$ model to be more "lossy" than the data, yet both codes seem to indicate less dissipation in the calculation than in the data. A possible effect that has not been explored is that the experiment used a dilute saline solution for the jet to measure concentration profiles as well as velocity profiles. Our calculations used water, so any differences in the physical properties would be manifested as discrepancies between the calculations and the data. Since we have no data on the uncertainty in the measurements, so we cannot make a quantitative assessment of the differences.

Another potential explanation for the observed response is sensitivity to the computational mesh. This will be explored in more detail in the discussion of similarity in the next section.

A last observation is that the FLUENT RNG $k-\varepsilon$ model resulted in a calculation closer to the data than either the CFDS-FLOW3D or FLUENT results already discussed. The RNG calculation was performed using the FLUENT $k-\varepsilon$ calculation as a starting condition, then changing the turbulence model and running the problem until convergence was achieved. As noted above in the comparison with data, this result was unexpected. The RNG model is thought to give less dissipation than the standard $k$ - $\varepsilon$ model, yet it appears to show just the opposite behavior here. The result is further unexpected because the turbulent jet was not expected to be a geometry in which the RNG model would show much difference from the standard model. It is designed more to treat other conditions such as curved streamlines and behavior near walls where the standard models do not work as well. No further sensitivities were performed with the RNG model for this study, but a more detailed assessment will be performed in the later mixing work.

\subsection{Similarity}

One of the key features of free turbulent jet behavior is the development of similarity profiles in the axial velocity in planes perpendicular to the jet axis once the development region is past. Figure 17 shows axial velocity profiles from both codes in several planes perpendicular to the jet axis and compares them to both data and classical models. While both codes developed similarity profiles, there are meaningful differences in the results.

One of the more obvious aspects of Figure 17 is that the CFDS-FLOW3D results developed similarity profiles quickly. The first set of results at ten diameters (10d) shows the same character as the remaining CFDS-FLOW3D profiles. Therefore, we conclude that the similarity was developed by ten diameters. Since the classical theory places the beginning of 


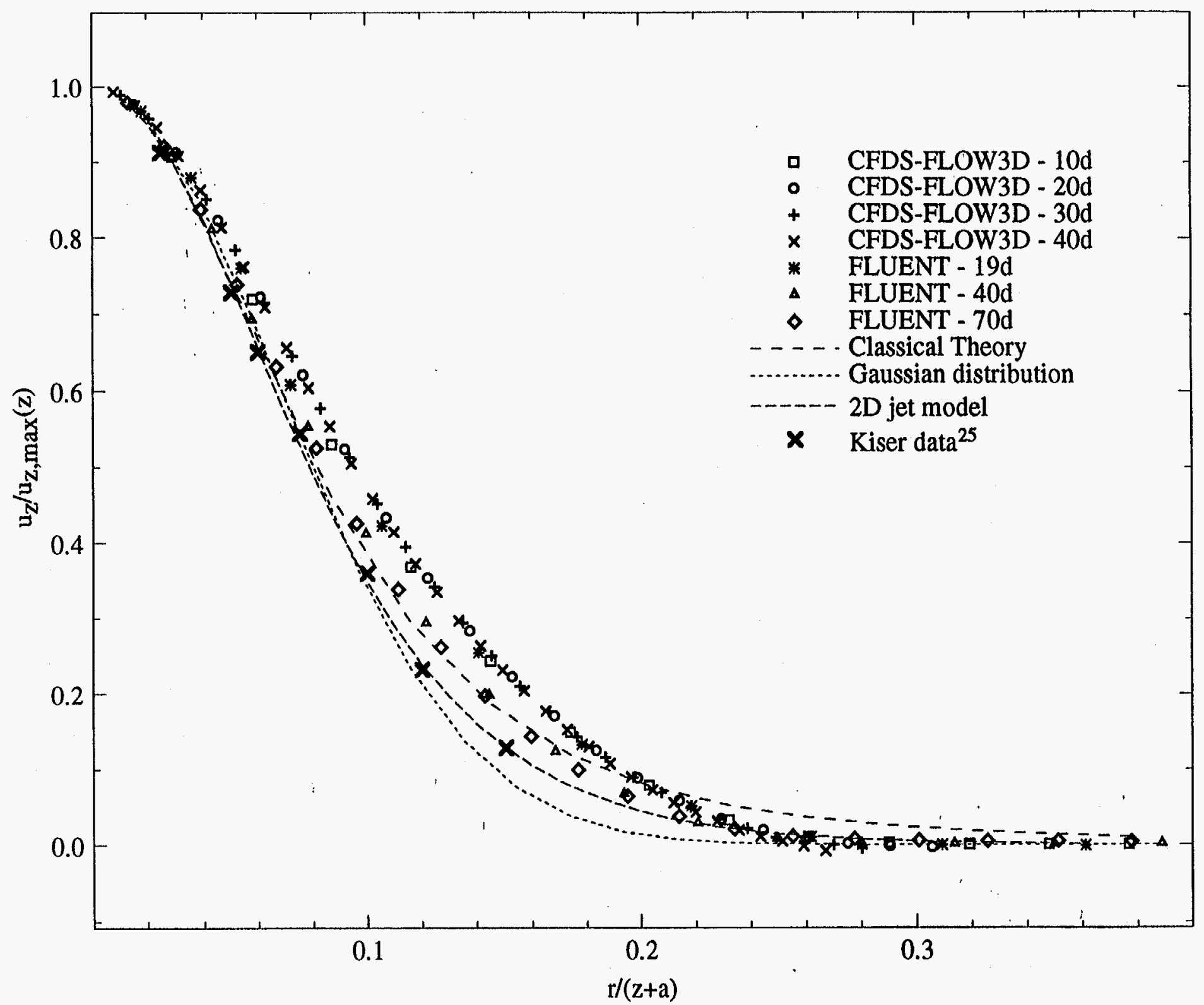

0
0
0
0
0
0
0
0
0
0
0

Figure $17 . \mathfrak{u}_{\mathrm{Z}}$ similarity profiles 
the similarity region at about six diameters, this result indicates agreement with the classical theory. The profile itself, though, is significantly broader than any of the approximate curves shown in Figure 17, and it is broader than the Kiser data. These results will be addressed shortly, but first, the FLUENT results need to be discussed.

The FLUENT calculation also showed the development of similarity profiles, but the results plotted in Figure 17 show that the similarity profiles took much longer to be established. The first data set plotted is at nineteen diameters, and it is significantly different from those shown for 40 and 70 diameters. While the similarity profiles took much longer to develop, the final result is in better agreement with both the classical theories and the Kiser data than the CFDSFLOW3D results. We think the differences seen here are more dependent on the mesh used for each model than they are on the individual codes.

Recall the discussion of Section 4.1, Basic grid setup. It was noted there that the FLUENT mesh used nearly twice as many nodes in the planes perpendicular to the jet axis than did the CFDS-FLOW3D mesh. The result of the finer nodalization in the FLUENT model is that a better discrimination of velocity variations away from the jet axis is possible. This is especially true far from the axis where the CFDS-FLOW3D mesh becomes extremely coarse. On the other hand, the coarse nodalization in the directions perpendicular to the axis left room for a finer nodalization along the axis. This resulted in the CFDS-FLOW3D model using nearly twice as many nodes in the axial direction as the FLUENT model. For the same reason that better discrimination of the radial behavior is seen in the FLUENT calculation, better discrimination of the axial behavior is seen in the CFDS-FLOW3D calculation.

Therefore, we expect that both codes would show the best aspects of each calculation were the nodalization in each model to be made finer where it is presently coarse.

\subsection{Spreading}

The spreading of the turbulent free jet is well characterized in the literature based on both an assumption of constant momentum flux and on measurements. For the round free jet, the spreading is expected to be linear in a stagnant ambient liquid region. The difficulty in gauging this performance with the CFD codes was in identifying the edge of the jet. In the same sense that very small perpendicular velocities could be used to determine asymmetries in the flow patterns that might not even be detected experimentally, a condition of zero axial velocity or radial velocity is hard to observe when the code can calculate very small numbers. In essence, the calculated velocity can be very small, but rarely zero. For this reason (since the first assessment attempt in this manner failed), an alternative approach was used.

As already noted, the velocities get very small near the edge of the jet. Kiser ${ }^{25}$ notes this problem and dismisses measured velocities along the edge of the jet as having too much uncertainty. Instead, he uses a set of half-widths to characterize the spreading of the jet. The velocity half-width is that location on the plane perpendicular to the jet axis at which the axial velocity is one-half its value on the axis in the same plane. Kiser's measured values of 
velocity half-width are compared to the values calculated by both FLUENT and CFDSFLOW3D in Figure 18. The results of both codes are quite close to Kiser's data and indicate the correct spreading rates, at least at the half-velocity point. A least squares fit of each of the three data sets plotted in Figure 18 shows the spreading angle of the velocity half-width to be:

$\begin{array}{ll}\text { FLUENT: } & 10.0^{\circ} \\ \text { CFDS-FLOW3D } & 10.4^{\circ} \\ \text { Kiser data } & 9.5^{\circ}\end{array}$

Another measure of jet spreading was devised in an attempt to capture the entire width of the jet. The momentum flux was integrated at the end of the development region, in this case at a plane five diameters from the nozzle in the FLUENT calculation. This value was taken as the constant momentum flux. The edge of the development region was selected as the reference point to avoid including losses, or changes in momentum, due to the initial development of the similarity profiles. At subsequent planes, the limits of integration across the jet were varied until the integrated momentum flux was equal to the value observed at the end of the development region, and the position was noted. This process was repeated along the entire length of the jet, and the results plotted as shown in Figure 19. Two observations can be made. First is that the profile is nearly linear, with a linear least squares fit drawn through the results as a reference. Second, the angle at which the jet spreads, according to the least squares fit, is $14.4^{\circ}$. This is in very good agreement with a generally accepted value of about $14-20^{\circ}$.

Another measure of the spreading of the jet is the volumetric entrainment rate as the jet dissipates. Using the limits of integration determined above from the constant momentum flux criterion, the volume flux across the jet was integrated and plotted in Figure 20. Again, it is obvious that the behavior is very close to linear, which is the expected result.

\subsection{Turbulence parameters}

As a measure of the performance of the turbulence models, both $k$ and $\ddot{\varepsilon}$ can be plotted in various regions of the jet. Figure 21 shows calculated values of $k$ and $\varepsilon$ along the jet axis from both FLUENT and CFDS-FLOW3D. In general, it shows the generation of a significant amount of turbulent kinetic energy and an associated dissipation rate, both of which decay as distance along the axis increases. The differences in the turbulent kinetic energy near the jet nozzle are caused by differences in the initial conditions used to start the calculations. The effects of these differences wash out by about twenty diameters from the nozzle, but this result shows that some care has to be taken if behavior near the nozzle is important. This might be the case when the calculations are extended to include temperature effects caused by the dissipation of energy in the fluid. 


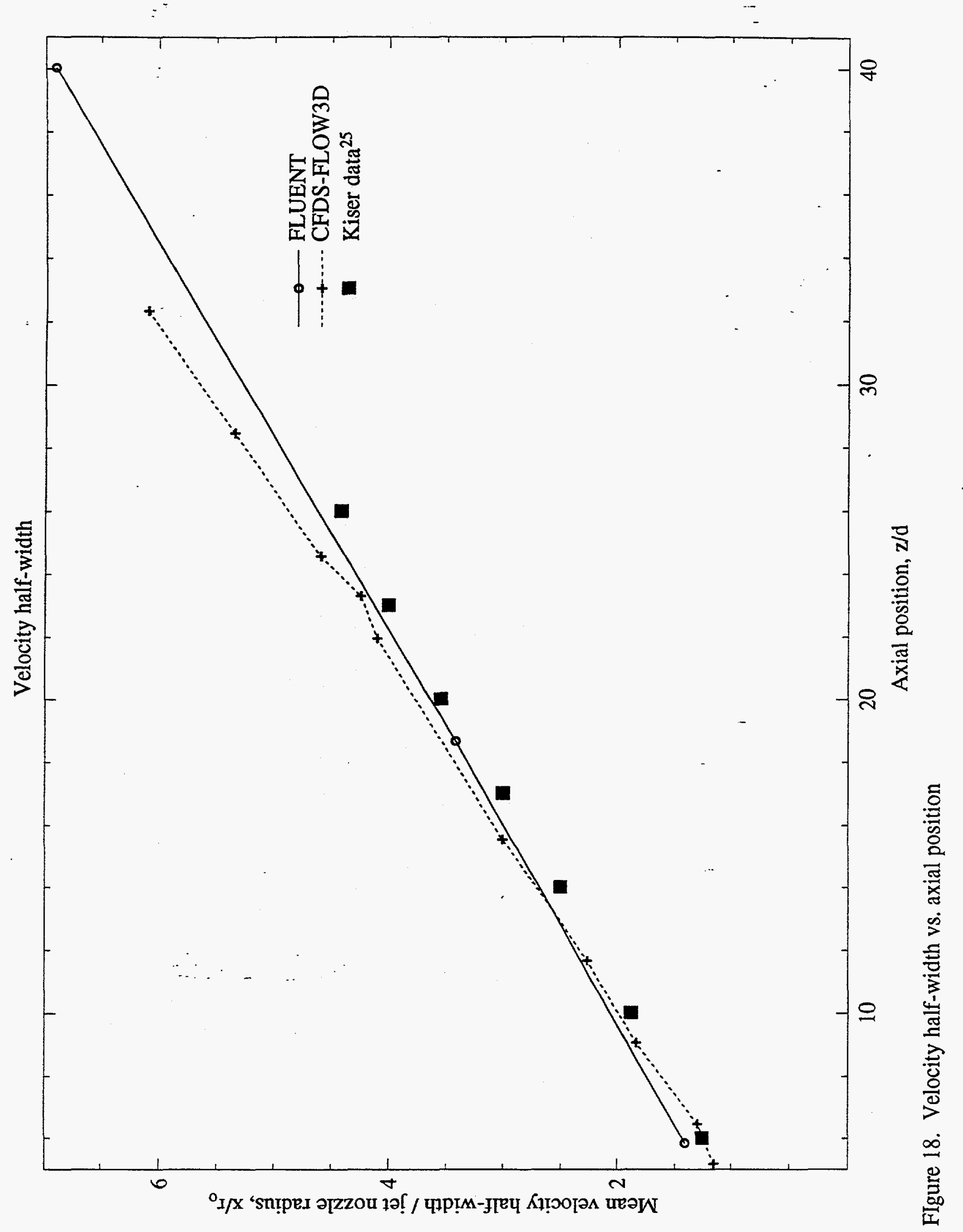


Jet Spreading

(based on constant momentum flux)

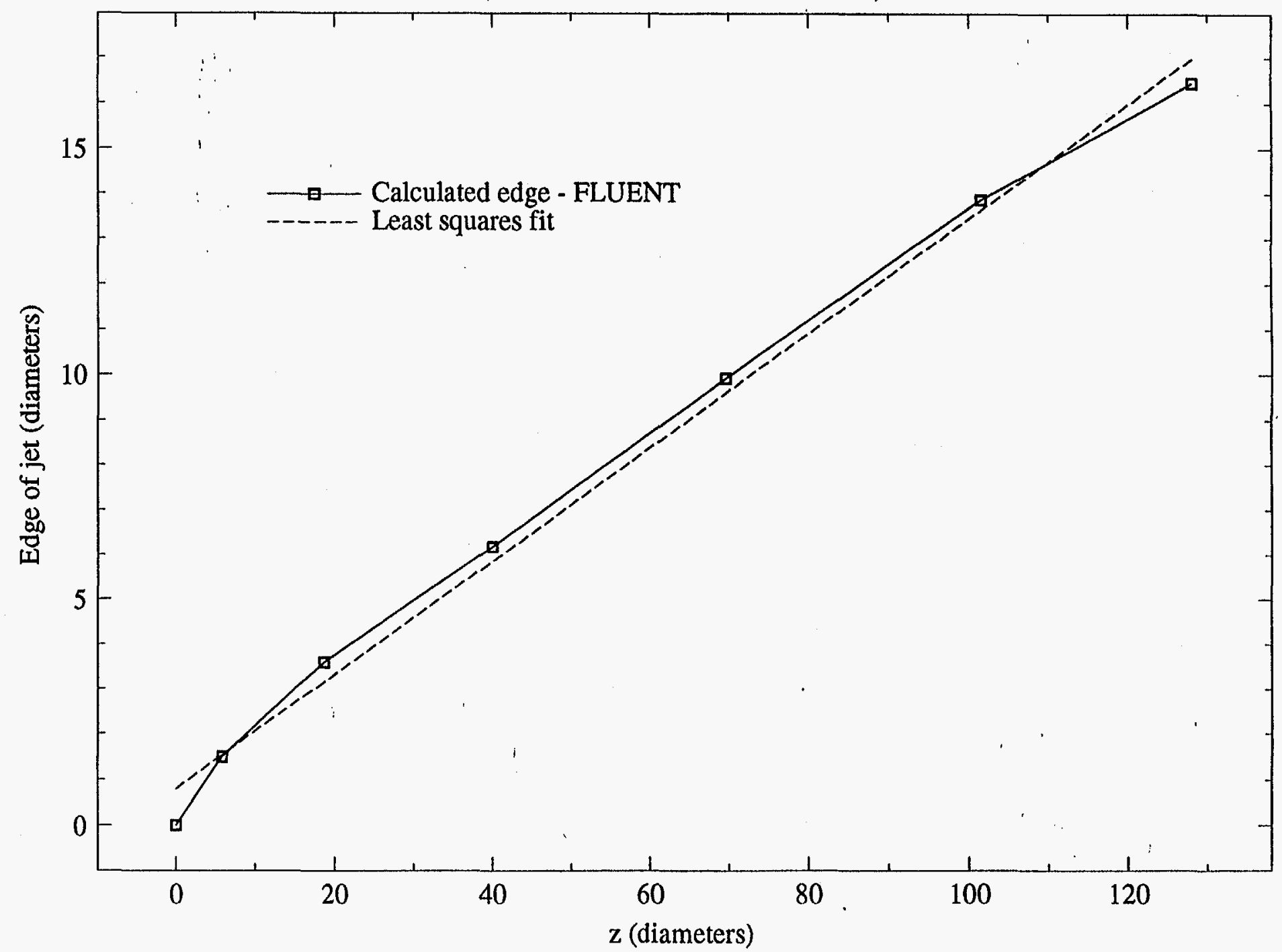

Figure 19. Jet spreading based on constant momentum flux. 


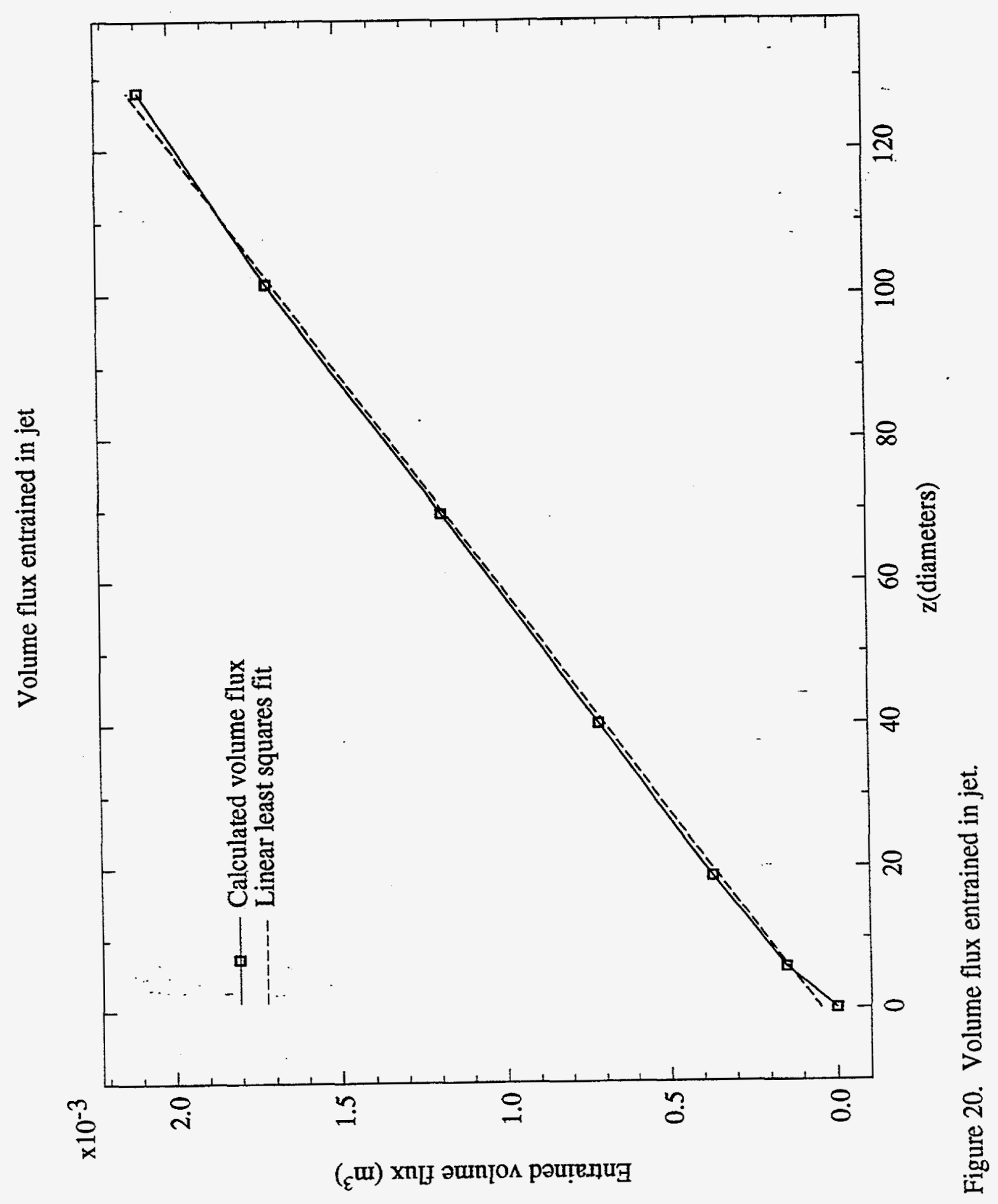




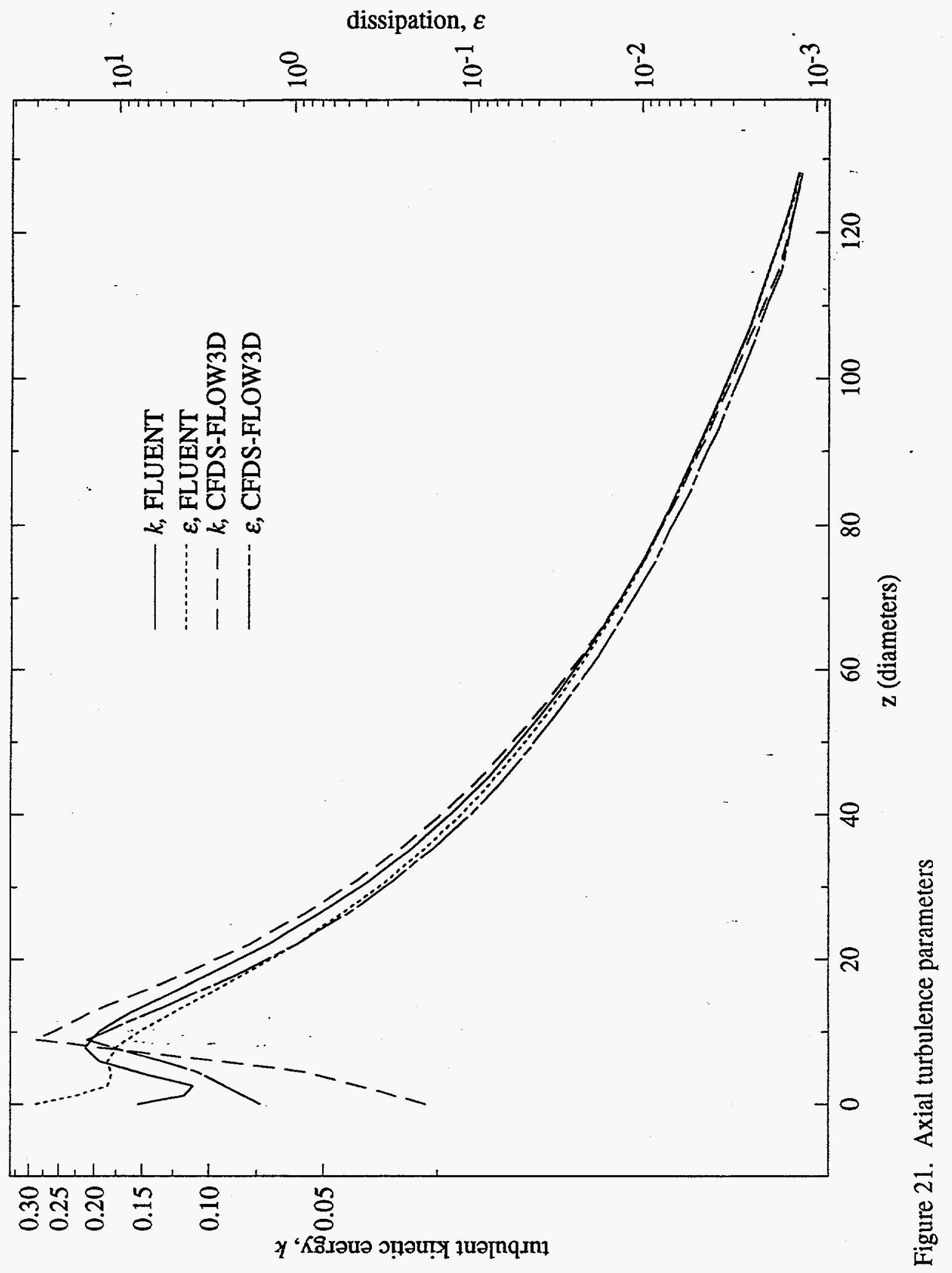


Radial profiles for the same two parameters are shown for the FLUENT and CFDS-FLOW3D calculations in Figure 22 at a plane about 60 diameters from the jet nozzle. The turbulent kinetic energy results are compared to data ${ }^{30}$ taken in an air jet at a similar location. The data were actually turbulence intensity measurements that we combined using Eq. (2.16) to infer a kinetic energy. Recall that neither code actually computes a turbulence intensity, so a direct comparison of code results and data was impossible.

The general character of the kinetic energy profile is similar between the calculations and the data, although neither code shows as steep a radial gradient as the data. The relatively flat behavior near the center of the jet is consistent with the classical observation that the kinetic energy is roughly constant across the turbulent region of the jet. The nature of the dissipation term is similar for the two codes, but there are no data against which to compare the results. These observations must remain qualitative since we have no direct data comparisons. It is expected that these parameters will become increasingly significant as we continue this project into a study of wall jets and ultimately tank mixing.

\subsection{Mesh sensitivity}

Mesh design has a significant impact on the performance of the CFD codes, both in terms of calculational accuracy and in terms of computer resources needed to perform the analysis. Of particular interest is the run time needed for a given calculation. Our desire is to use the coarsest mesh possible to minimize the time needed for calculations. However, computational accuracy is affected by the size and spacing of the nodes used to represent the physics of the flowing system.

Figure 23 compares the normalized axial velocity calculation for several runs. A FLUENT and CFDS-FLOW3D run each using about 20,000 nodes are shown to give similar results. In addition, a FLUENT run using the same geometry and boundary conditions, but only 2700 nodes, is shown. The noding scheme is the same for both of the FLUENT runs shown, but the cells in the 2700 -node model are approximately twice as big in each linear dimension as those in the 20,000-node model. Clearly, the results are different. This indicates that the 2700-node model is not fine enough to capture the correct spatial behavior of the system, even though the results satisfied the FLUENT convergence criteria. Our conclusion is that the mesh cells are too big to allow the code to properly represent the flow. A similar calculation with CFDS-FLOW3D with a more detailed model which used 60,000 nodes instead of 20,000 showed that the results were very nearly the same as the 20,000-node case. In this case, we don't see a sensitivity to the grid spacing and conclude that the 20,000-node case is fine enough to capture the correct flow behavior. We expect that both conclusions about the adequacy of the mesh are true for both codes, and that the sensitivity calculation does not need to be run separately for both codes. These results will be useful in estimating the maximum acceptable cell dimensions for subsequent calculations in the mixing tanks. 


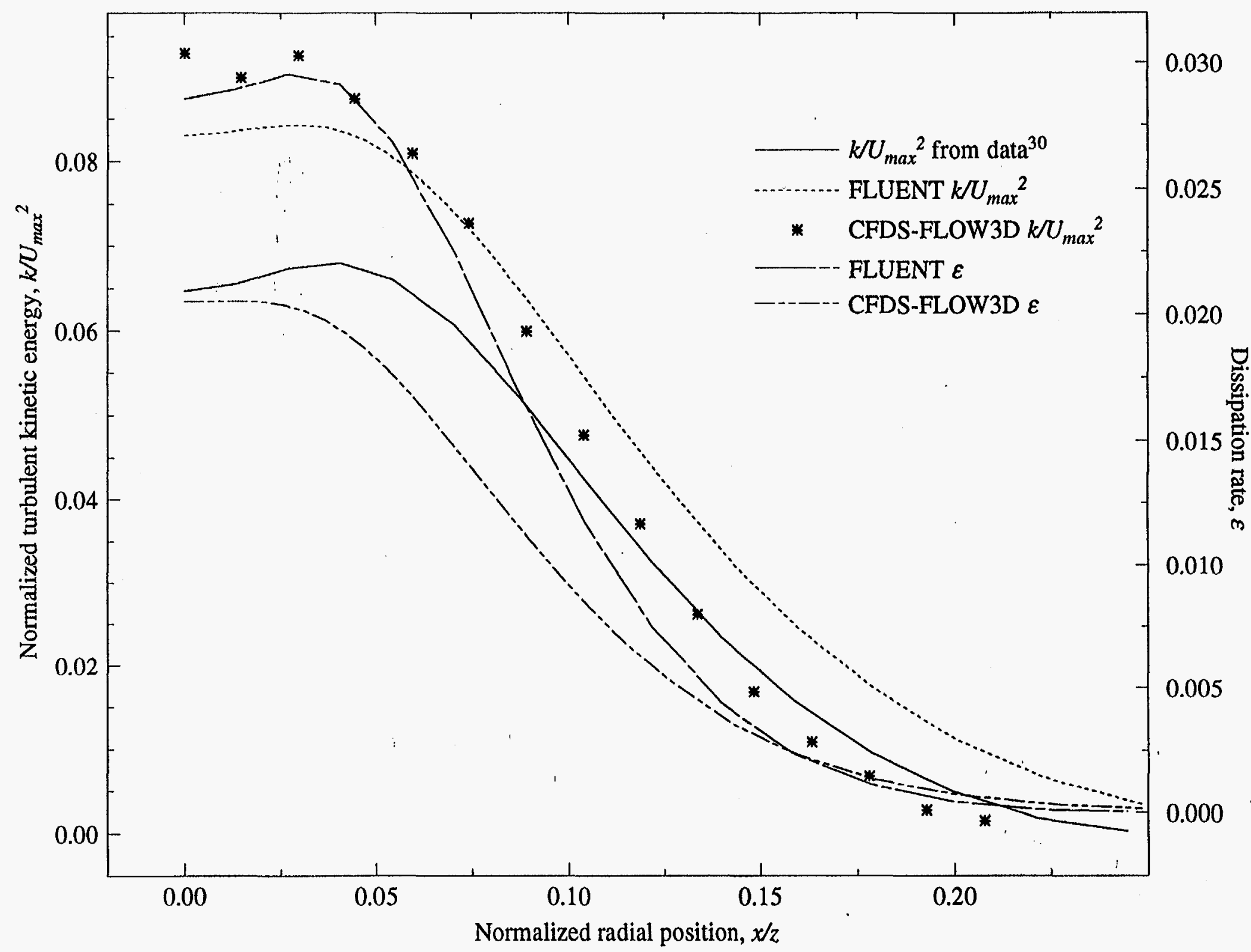

Figure 22. Radial turbulence parameters at 60 diameters. 
WSRC-TR-95-0170

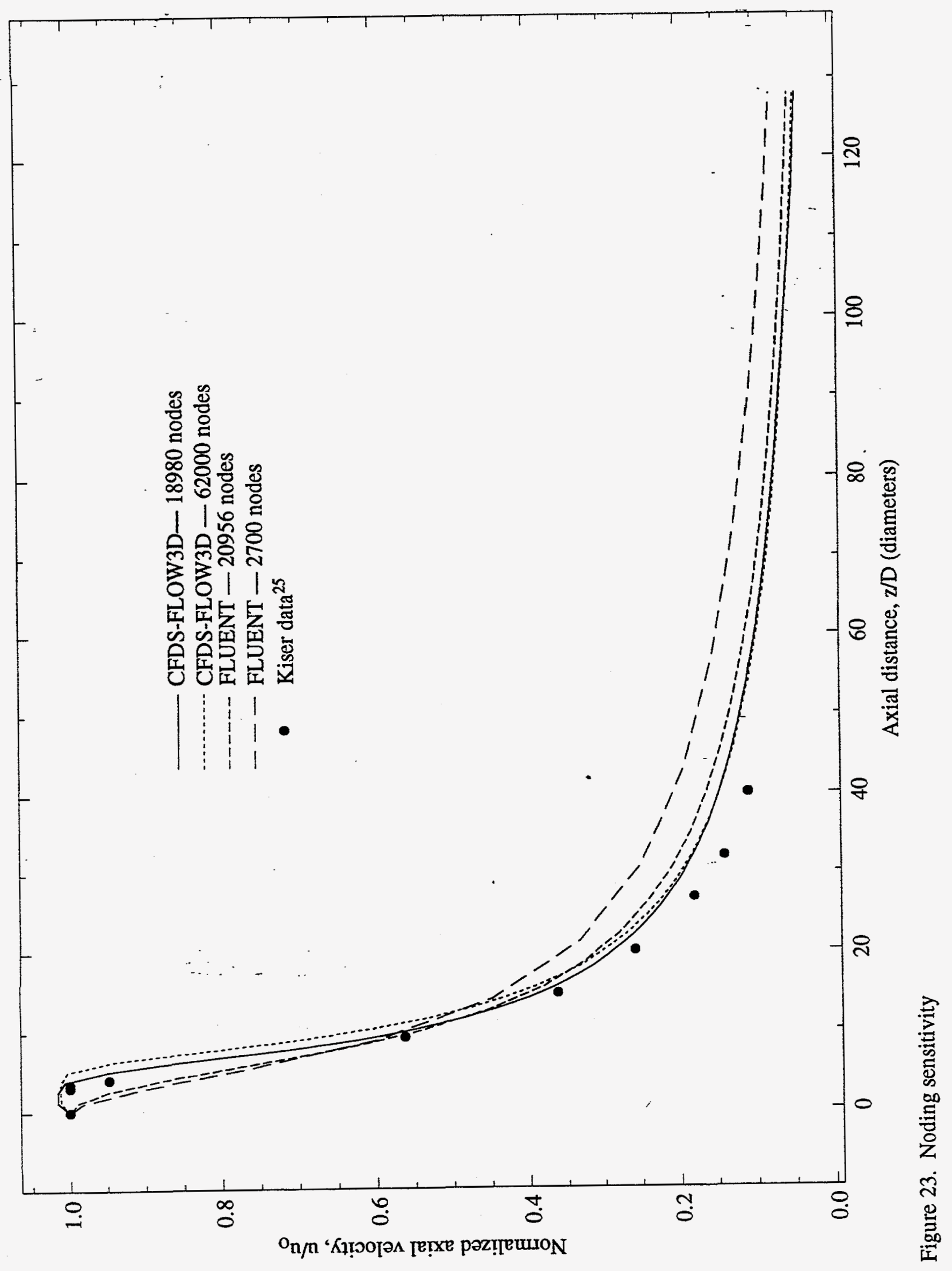




\section{Conclusions}

The ability of two CFD codes, FLUENT and CFDS-FLOW3D, to calculate the significant physical characteristics of an axisymmetric free turbulent jet of water was shown through comparisons with data and classical jet theory. Both codes performed well and were considered acceptable for further applications to tank mixing analyses at SRS. No significant differences between the two codes were identified.

Specific conclusions associated with detailed comparisons between calculations and data are as follows:

1. Axial velocity along the jet centerline decreases inversely with $x / d$ when the distance from the nozzle is greater than six diameters.

2. Velocity profiles of axial velocity along a direction perpendicular to the jet axis are similar within the fully turbulent region.

3. Total momentum flux of the fluid in the jet is approximately constant perpendicular to the jet axis under isothermal flow conditions.

4. The angle contained between the jet flow axis and the zero axial velocity location along the edge of the jet is constant for distances greater than six diameters away from the jet nozzle.

5. Turbulent eddy viscosity, $v_{t}$, is a function of local axial velocity and spreading angle, $\alpha$, based on the two-dimensional similarity relationships. This behavior is supported in the literature.

6. Volumetric entrainment in the jet is linear with axial distance and consistent with classical jet theory.

7. Sensitivity to the model node density will have to be determined when mixing calculations are performed for the HLW waste tanks.

8. Both FLUENT and CFDS-FLOW3D give similar results for the parameters tested. FLUENT output capabilities are somewhat more convenient and flexible than CFDSFLOW3D.

\section{Nomenclature}

Variables 
A

$c$

$c_{p}$

$C 1 \varepsilon, C 2 \varepsilon, C 3, C \mu$

$d$

D

$f, h$

$h$

H

F

$g$

$G b$

$G_{k}$

$J$

$k$

$\ell$

$m$

$p$

$r$

$S$

$t$

$T$

$u_{i}, u_{x}$

$u_{o}$

$\boldsymbol{u}$

$x$

\section{Greek symbols}

$\begin{array}{lll}\alpha & & \\ \delta_{i j} & & \\ \varepsilon & & \\ \phi & & \\ \Phi & \ddots & \\ \lambda & & \\ \mu & & \\ \nu & & \\ \rho & & \\ \sigma & & \\ \sigma_{k}, \sigma_{\mathcal{E}}, \sigma_{h} & \\ \tau & & \\ \xi_{2} & \end{array}$

constant

constant

specific heat at constant pressure

empirical constants

jet nozzle diameter

diffusion term

arbitrary functions

enthalpy

total enthalpy

momentum source term

acceleration of gravity; arbitrary function

generation rate of turbulence from buoyancy

rate of production of turbulent kinetic energy

mass flux of species

turbulent kinetic energy; mixture thermal conductivity (Eq. 2.20)

mixing length

mass

pressure

radial dimension

mass source term

time

temperature

scalar component of velocity

jet nozzle exit speed

velocity

component of position vector

jet expansion angle

Kronecker delta

dissipation of turbulent kinetic energy

arbitrary scalar

volume flux

thermal conductivity

dynamic viscosity

kinematic viscosity

density

shear stress tensor

"Prandtl" numbers

stress tensor

nondimensional position perpendicular to jet axis 


\section{Superscripts}

$l$

$p, q, r, s_{21}$

$t$

\section{Subscripts}

$1 / 2$

$0, o$

$h$

$i, j$

$i^{\prime}, j^{\prime}$

$l$

$m$

$\max$

$o p t$

$r$

ref

$s$

$t$

$z$ laminar

free exponent

turbulent

half-width position

issuing value at jet nozzle

enthalpy

indices representing each of the component directions

index denoting species

dummy index for summation over component directions

mixing, as in mixing length; mass

maximum value, generally associated with value on jet axis

optimum or maximum

radial component direction

reference value

ambient fluid

turbulent

axial component direction

\section{References}

1. Process Requirements. $241-82 \mathrm{H}$ Control Room (U), Savannah River Site, WSRC-IM91-63, February, 1995, PR 3.1.1.3, 3.1.1.2, 3.1.1.4, 3.3.1.3.

2. Dimenna, R. A. memorandum to M. R. Poirier, "Mixing Analysis Program," SRTEMS-950006, April 7, 1995.

3. Bird, R. B., Stewart, W. E., and Lightfoot, E. N., Transport Phenömena, John Wiley \& Sons, Inc., New York, 1960.

4. Govier, G. W. and Aziz, K., The Flow of Complex Mixtures in Pipes, Van Nostrand Reinhold Company, New York, 1972.

5. Aris, R., Vectors, Tensors, and the Basic Equations of Fluid Mechanics, Dover Publications, Inc., New York, 1962.

6. Kays, W. M. and Crawford, M. E., Convective Heat and Mass Transfer, Second Edition, McGraw-Hill, Inc., New York, 1980.

7. Hinze, J. O., Turbulence, McGraw-Hill, Inc., New York, 1975.

8. Schlicting, Boundary Layer Theory, McGraw-Hill, Inc., New York, 1968.

9. Tatterson, G. B., Fluid Mixing and Gas Dispersion in Agitated Tanks, McGraw-Hill, Inc., New York, 1991. 
10. Kotsovinos, N. E., "A note on the conservation of the axial momentum of a turbulent jet," J. Fluid Mech. 87, 1978, pp. 55-63.

11. List, E. J., "Turbulent Jets and Plumes," Ann. Rev. Fluid Mech., 14, 1982, pp. 189 212.

12. Rajaratnam, N., "Theory of Turbulent Jets," in Handbook of Fluids in Motion, N. P. Cheremisinoff and R. Gupta, Eds., Ann Arbor Science, 1983, pp.-251-278.

13. Townsend, A. A., "The mechanism of entrainment in free turbulent flows," $J$. Fluid Mech., 26, 1966, pp. 689-715.

14. Townsend, A. A., "Entrainment and the structure of turbulent flow," J. Fluid Mech., 41,1970, pp. 13-46.

15. Townsend, A. A., The Structure of Turbulent Shear Flow, 2nd Ed., Cambridge University Press, 1976.

16. Schneider, W., "Flow induced by jets and plumes," J. Fluid Mech. 108, 1981, pp. 5565.

17. Schneider, W., "Decay of momentum flux in submerged jets," J. Fluid Mech. 154, 1985, pp. 91-110.

18. Zauner, E., "Visualization of the viscous flow induced by a round jet," J. Fluid Mech. 154, 1985, pp. 111-119.

19. Cho, J. R, and Chung, M. K., “A $k-\varepsilon-\gamma$ equation turbulence model," J. Fluid Mech. 237, 1992, pp. 301-322.

20. Arpaci, V. S. and Larsen, P. S., Convection Heat Transfer, Prentice-Hall, 1984.

21. Rodi, W., "Turbulence Models for Environmental Problems," in Prediction Methods for Turbulent Flows, W. Kollman, ed., Hemisphere Publishing Corporation, 1980, pp. $259 \mathrm{ff}$.

22. Tennekes, H, and Lumley, J. L., A First Course in Turbulence, The MIT Press, 1972.

23. Lumley, J. L, "Second Order Modeling of Turbulent Flows," in Prediction Methods for Turbulent Flows, W. Kollman, ed., Hemisphere Publishing Corporation, 1980, pp. $1 \mathrm{ff}$.

24. FLUENT User's Guide, Ch. 13, FLUENT Incorporated, Lebanon, NH, June 1993.

25. Kiser, K. M., "Material and Momentum Transport in Axisymmetric Turbulent Jets of Water," A. I. Ch. E. Journal, 9, May, 1963, pp. 386-390.

26. Forstall, W. and Gaylord, E. W., "Momentum and Mass Transfer in a Submerged Water Jet," J. Appl. Mechanics, 22, 1955, pp. 161-164.

27. FLOW3D Release 3.3. User Manual, Computational Fluid Dynamics Services, AEA Industrial Technology, Harwell Laboratory, Oxfordshire, UK, June 1994.

28. Abramovich, G. N., The Theory of Turbulent Jets, MIT Press, Cambridge, 1963.

29. Rushton, J. H., "The Axial Velocity of a Submerged Axially Symmetrical Fluid Jet," AIChE Journal, 26, No. 6, November, 1980, pp. 1038-1041.

30. Wygnanski, I. and Fiedler, H., "Some measurements in the self-preserving jet," $J$. Fluid Mech., 38, 1969, pp. 577-612. 
Appendix A

Mixing Analysis Program (Reference 2) 
WESTINGHOUSE SAVANNAH RIVER SITE

INTEROFFICE MEMORANDUM

April 7, 1995

CC: J. N. Brooke, 719-4A

J. E. Marra, 703-H

M. R. Buckner, 773-42A

D. R. Muhlbaier, 786-5A

B. J. Hardy, 773-41A

J. R. Pelfrey, 773-42A

N. Hassan, 773-A

W. L. Tamosaitis, 773-A

E. D. Lee, 241-152H

G. A. Taylor, 703-H

S. Y. Lee, $773-42 \mathrm{~A}$

J. P. Wood, 676-1T

TO:

M. R. Poirier, 676-T, 5-6000

FROM:

R. A. Dimenna, 773-42A, 5-5300

RE:

Mixing Analysis Program

The question of characterizing the mixing behavior in the high level waste storage and process tanks has long been recognized as a difficult one. Traditional phenomenological models and rules-of-thumb are not precise enough to justify running the jet mixing pumps at anything less than continuous full speed operation, yet such an operational program has the potential of causing temperature limits in the tanks to be exceeded. Therefore, a more detailed tank mixing analysis that can support less aggressive pump operations is desirable.

The attached analysis program describes a step-by-step approach to developing a liquid mixing analysis capability for the high level waste storage and process tanks. It is centered about operational questions posed by you on behalf of HLWE and ITP. The tasks described in this program couple the development of a basic analytical capability with applications to these important site issues. The program is designed to provide useful rësults as individual pieces of a general capability are developed, but it does not represent all of the development necessary to address every mixing issue that will arise in the future. It will provide the basic tools needed to address those future issues, though, and continuing development will address additional phenomena as extensions to the capability described here.

The work described in the attached analysis program is partially completed. The first draft report addressing turbulent free jet behavior was completed and sent to you for comments on March 10, 1995. Work began on the second and third tasks, the wall jet analysis and vortex analysis, during the same time that the draft report on the submerged free jet was being prepared. Some results from that partially completed work will be reported to you in the form of a memorandum. 
M. R. Poirier

SRT-EMS-950006

You will note that the schedule for completing the remainder of the attached program is quite aggressive and includes writing many reports. Our current feeling in EM\&S is that it is appropriate to push this CFD capability as hard as possible so that we can establish and document this capability quickly. To that end, we developed a schedule that is tight but doable.

Currently, our work is limited to addressing reviewer comments on the turbulent free jet draft report. Several reviewers, including yourself, have returned comments, and all comments will be addressed in the final report. With the exception of completing that report, all work on this project was stopped in mid-March.

Attachment as stated 


\section{Attachment}

\section{Analysis of Fluid Phenomena and Mixing in High Level Waste Tanks}

\section{Introduction}

The development of a computational mixing analysis capability at the Savannah River Site (SRS) was initiated to provide operational guidance for the High Level Waste (HLW) storage and process tanks. Specifically, the High Level Waste Engineering (HLWE) - In-Tank Precipitation (ITP) organization requested information on how to operate the jet mixing pumps in Tank 48 to comply with tank temperature limits while maintaining adequate mixing in the tank. Traditional phenomenological models and rules-of-thumb indicated that the pumps should be run continuously at full speed, but that had the potential of overheating the tanks. A more detailed analysis with computational fluid dynamics (CFD) codes was proposed to determine a different pump operational program that would optimize the mixing performance and result in less energy being deposited into the tank. Such would be the case if all four pumps in Tank 48 did not have to run continuously, or if they could run at less than full speed. That would reduce, and perhaps eliminate, the need for additional heat removal capability to keep the tank in compliance with operating temperature requirements.

Tank 48 is a Type IIIA tank, one of five types of large tanks used to store high level waste at SRS. From a liquid mixing point of view, it is typical in its geometry of the approximately 1million gallon tanks having a center column, but its contents are easier to characterize (in a computational sense) than those of the sludge tanks. Tank 48 contents are principally liquid with a varying concentration of suspended solids. The tank contents are mixed by four submerged pumps which rotate slowly while discharging opposing high speed jets of tank fluid. These jets are designed to maintain sufficient liquid motion to keep the contents homogeneous, thus ensuring that chemical reactions and washing operations are completed, and that solids remain uniformly distributed throughout the tank. The goal of the computational mixing analysis program is to determine the appropriate values for tank operational parameters which will optimize the ITP process. This will be accomplished by assessing their impact on tank mixing, heat generation, and entrainment of air into the liquid space. Similar questions have been raised about the performance of slurry pumps in suspending and mixing sludge in the Extended Sludge Processing (ESP) tanks such as Tank 42. Operational parameters beyond the initial set, or parameters specific to ESP tanks, may be identified in the course of completing the mixing analysis.

The physical characteristics of the HLW storage and processing tanks at SRS makes a detailed analysis challenging. The tanks encompass a range of geometries, materials, and operational processes that extend beyond the current state of the art of mixing technology. The performance of any specific part of the system depends on incoming flow stream 
compositions as well as fluid behavior within the tank. Discharge stream composition must meet strict limits as well to satisfy the requirements imposed by downstream processes. A mixing analysis must be able to treat not only the effects of large physical dimensions of the tanks, but also the smaller scale effects such as jet nozzle diameter or specific injection sites for incoming streams. The operational parameters thought to be useful for optimizing process performance are those that directly affect mixing behavior, viz.:

1. Pump flow rate, including possible on-off cycles.

2. Pump elevation.

3. Pump rotation rate.

4. Batch size.

5. Tank liquid level.

The combination of these parameters, as well as the large physical size of the waste tanks and the hazardous nature of the contents makes a computational approach more attractive than an experimental one in terms of both time and cost to complete the work.

The method by which mixing performance or temperature compliance will be observed within Tank 48 is also a mixing issue, just as much as characterizing flow patterns within the tank. The mixing behavior affects temperatures in the tank through the distribution of energy in a manner similar to momentum or material concentration. Uniformity of tank temperature is as significant an issue as uniformity of material composition or density in the form of build up in local regions of the tank. The interpretation of local temperature measurements as an indicator of bulk or mixed temperature is an issue that can be addressed through the computational capabilities being developed.

The overall question of mixing performance in the HLW tanks was narrowed somewhat when three specific questions were posed by HLWE and IWT:

1. At what liquid level does a vortex formed from the pump operation begin to draw air into the liquid region of the tank?

2. At what liquid level does a pump discharge jet aimed parallel to or toward the liquid surface break the surface and begin to entrain air into the liquid region, the -so-called "rooster tail" behavior?

3. At what tank liquid level does the liquid flow induced by the pumps climb the tank wall, break the liquid surface and begin to entrain air into the liquid region?

These questions concerning ITP operations in Tank 48 are an integral part of the overall mixing issue, but they are less complex than the global mixing behavior. They involve the same basic driving phenomena that affect the overall mixing, so they represent a convenient set of issues that will be answered as interim goals in the analysis of tank mixing. 


\section{Methods Development}

The Engineering Modeling and Simulations (EM\&S) group of the Applied Technology Section (ATS) began developing a liquid mixing analysis capability when the In-Tank Precipitation (ITP) organization asked us to determine the minimum pump power needed to mix the contents of Tank 48. The Tank 48 issues were a good starting point for this work because all of the HLW mixing phenomena were present without the added complication of a sludge layer. After a joint review of computational fluid dynamics (CFD) codes, ITP and EM\&S selected and obtained two CFD codes that were applicable to our liquid mixing problems, CFDS-FLOW3D and FLUENT. Both of these codes were evaluated and applied by $E M \& S$ in the analysis of the turbulent free jet.

The analytical approach recommended here addresses the behavior of the liquid space in Tank 48. At the outset the problem can be reduced to establishing the performance of the computational codes when calculating the characteristic behavior of turbulent free jets. The specific parameters to be assessed will be discussed below. With the turbulent free jet capability established, the calculations can proceed naturally to wall jets, rotating jets, free surface phenomena, wall interactions when the jet impinges on a vertical wall, and the interactions of flow patterns established by independent jets. Other phenomena needed for the tank mixing analysis such as particle suspension and setting rates, jet cleaning radius as sludge is suspended from the bottom of the tanks, or tank temperature behavior as the effects of energy transfers to and from the tank are included can be added incrementally.

The questions concerning air entrainment as a function of liquid level will be treated without addressing the air mixing issue per se. They are affected first by the behavior of the turbulent jet issuing from the pumps, and second by the free surface behavior at the interface between the liquid and air (or gas). The phenomena leading up to an air entrainment condition will be analyzed, but the calculations will not be extended beyond that point.

The work described in this analysis program is equally applicable to ITP and ESP. The only significant difference between the two applications is the influence of sludge on fluid behavior and fluid properties in ESP tanks. The presence of sludge doesn't change the fundamental physics per se, but it requires that shear stresses induced by the fluid jet passing over a sludge layer be represented well enough to calculate sludge suspension. This particular phenomenon is not present in the ITP applications. Depending on customer needs, a treatment of sludge behavior could be inserted into the proposed program or added at the end as a natural extension to the current work.

The development described here deliberately uses two different computational codes to test against each other. The purpose of this approach is to ensure the adequacy of both codes in the absence of validation data. While some data that provide approximate descriptions of 
fluid properties and behavior in the HLW tanks have been identified, none satisfy the level of detail or precision needed to adequately validate a computer code. Some more general data are available from outside organizations, including appropriately scaled slurry settling data from Pacific Northwest Laboratories (PNL), but none have been purchased or contracted by SRS. Therefore, the less satisfactory approach of comparing one computer code against another has been taken. This is a significant improvement over using only a single code with no independent validation, and the difference in cost between licensing a single code or two codes is small.

\section{Analysis}

The following summary describes the development of a tank mixing analysis capability through applications to current operational issues. The analysis progresses in steps to address specific customer questions while continually developing a capability to answer more complex tank mixing issues.

The topics to be addressed were determined from the questions posed by HLWE and IWT. They deal with phenomena important to mixing behavior in the tanks, and in this regard represent a consistent approach to developing a general mixing analysis capability. They are:
1. Jet behavior
2. Vortex behavior
3. Wall climbing
4. Tank mixing

An important part of the analysis will be determining the effects of variations in several fluid, operational, and modeling parameters. The basic operating parameters to be tested in this analysis were discussed above. Their impact will be felt even in the early stages of the analysis as the influence of boundary conditions on fundamental jet behavior is evaluated. Their effects will be coupled with those of basic physical models, including: turbulence models and the associated transport of turbulent momentum; fluid viscosity for Newtonian fluids; and non Newtonian behavior and the rheological model used to represent it. Each of these parameters is expected to have a significant impact on the fluid behavior in the tanks, but there are few däta with which to validate the calculated results. Therefore, the principal validation will come from comparisons between independent calculations. For this reason, two computer codes are being employed, CFDS-FLOW3D and FLUENT. This approach provides a significant measure of assurance beyond that of using a single code.

\section{Jet behavior}

Jet phenomena are basic to our understanding of fluid behavior in the tanks. The fluid jets forming the discharge of the pumps affect vortex formation, rotational forces, flow patterns 
that would break through the free surface or climb the walls, and both local and bulk flow patterns that induce mixing in the tanks. With regard to assessing CFD code performance, three jet configurations are identified. They are:
a. Submerged free jet - no mechanical boundaries affecting the flow.
b. Bounded jet - bounded on the lower side by the floor of the tank.
c. Surface jet - bounded on the upper side by the tank free surface.

1a. Submerged free jet. With the exception of completing the documentation, work has -already been completed on the submerged free jet problem with both CFDSFLOW3D and FLUENT. This included validating computational results against data for axial velocities both on and off the jet axis. Data were taken from the open literature. They were limited to water since there are very little data available for jet behavior in other liquids. Parametric variations included an initial test of the impact of the different turbulence model options in the two codes, but they did not include variations in the working fluid. This work will establish the basic capability of each of the codes to calculate a free jet.

1b. Bounded jet. Since the pumps in Tank 48 (as well as the other tanks) are located near the bottom, the calculation of wall jets is of next importance. The work with free jets will be extended to wall jets, again with water as the working fluid. The impact of the various turbulence model options is expected to be greater in this study, since the standard $\mathrm{k}$ $\varepsilon$ model is thought to work pretty well for the free jet, but not so well for the wall jet. Other special models such as near-wall turbulence models may become important in this part of the analysis.

The influence of pump operating parameters will begin to be felt in this portion of the analysis through the placement of the jet relative to the bounding wall. This sensitivity will help determine part of the influence of pump elevation. Other parametric variations will be extended in this phase of the analysis, especially changes in viscosity for a Newtonian fluid. The computational impact of this change on both free and wall jet behavior will be determined, but comparisons with data may not be possible because of the lack of data. A further extension to non Newtonian behavior will be completed by assessing the effect of a power law model.

1c. Surface jet. The surface jet problem includes all of the parameters mentioned so far plus one more, the free surface. Since the study of vortex behavior will address the basic free surface capability of the codes, the surface jet analysis will be postponed until the vortex work is done. Once the free surface models are understood, we will begin the surface jet analysis to address the "rooster tail" question, with one caution. We do not feel that the computational codes have the ability to calculate rates of air entrainment correctly. Therefore, even though the specific questions being addressed in this work involve 
air entrainment into the tank liquid region, the calculations will be taken up to, but not past, the point of incipient air entrainment.

The surface jet work will start with a horizontal jet below the free surface, but close enough to the surface to affect its behavior. Literature data are available for benchmarking the calculation of water jets in this geometry. The additional case of a jet pointed toward the free surface from below will be analyzed with the intention of calculating the deformation in the free surface up to, but not including, surface breakthrough. We don't expect that any CFD code can calculate the correct rate of air entrainment across the free surface.

\section{Vortex behavior}

The vortex behavior work will be the first to directly address one of the three HLWE questions, viz. the liquid level at which a vortex formed by the pump suction begins to draw air into the liquid region of the tank. This is inherently a free surface problem and requires the CFD package to have a specific free surface solution capability. Both FLUENT and CFDS-FLOW3D use a volume-of-fluid (VOF) technique to compute the location and shape of the free surface.

An analysis of vortex formation in the tank will be performed in parallel with the jet work described above. It will begin with a simple analysis to show formation of a vortex in a tank draining from a single hole centered in the bottom. The analysis will then be extended to a centered jet pump within the tank drawing suction from the bottom of the pump. Once that capability is established, a rotating jet boundary condition capability will be added to the codes through user FORTRAN options. Much of this modeling has already been completed for use in FLUENT. Other operational parameters will be addressed through the imposition of boundary conditions. The most important is probably the effect of tank liquid level which is imposed through the location of the free surface. Pump speed and pump elevation are other potentially significant variables in this analysis.

The initial analysis of vortex behavior will not include surface breakthrough or wall climbing, but it will include the influence of turbulence models and fluid rheological property models, as well as jet rotation from the pumps. The analysis will start with two-dimensional models, and will be extended to three dimensions only after some sensitivities have been characterized. The reason for delaying the extension to three dimensions is the significant computer run times that are anticipated.

\section{Wall climbing}

The wall climbing problem, the third of the HLWE questions posed, is simply the redirection of the jet flow when the jet impinges on the tank wall. This is expected to be one of the easier problems to solve, since the codes have already been shown to represent this type of behavior 
quite well. Because of this, and because HLWE considers it the lowest priority of the three basic phenomenological questions posed, this analysis will follow that of the vortex and the surface jet. A short analysis demonstrating the behavior of the jet when it impinges on the tank wall will be completed, including a parametric evaluation of the influence of material properties and an evaluation of the effect on the tank free surface. In addition, the effect of pump and tank operations on wall climbing behavior will be investigated, including such parameters as pump speed, pump rotational speed, pump elevation, tank volume (liquid level), and perhaps pump location.

\section{Tank mixing analysis}

Tank mixing analysis is a combination of the individual effects described above with the behavior of the fluid as a whole in the large mixing tank. The problem is difficult even without considering the real complexities of the internal structures of the mixing tanks, largely because of the wide range of length scales that must be treated in the coupled analysis.

The characteristic dimensions necessary to represent the whole tank as a single model are too limiting to allow a reasonable grid to be developed. The pump nozzle diameter establishes a small scale requirement to resolve the jet behavior as the fluid emerges from the pump. This dimension is about 1.5 inches, so the small scale model dimension should be no greater than about one-quarter of that, or about 0.4 inches. At the same time, the tank diameter is about 85 feet, and this entire span must be covered with a computational grid. Requirements on acceptable aspect ratios for computational cells and acceptable rates of expansion for adjacent cell dimensions would lead to an unacceptably large number of computational cells were the entire problem to be modeled as a single region. In this case, we are defining unacceptably large as a problem that is too big to fit on an SRS computer and run in a reasonable amount of time. Therefore, the tank mixing analysis has to be treated as at least two regions.

The first region is that one close to the pump. In this region, the characteristic dimension is the pump nozzle diameter. Jet spreading is calculated by starting with this small dimension and gradually expanding the computational mesh until the edge of the region is reached. The edge will be defined by the ability to establish a useful set of boundary conditions with which an adjacent region can be driven while keeping the size of the computational model reasonable. The other region is the rest of the tank, its characteristic dimension the tank diameter. By employing the outer dimension of the first region as the smallest dimension of the second region, the entire span of the tank can be modeled without an inordinately large number of cells. Therefore, the analysis will treat the two regions separately, with results from the region close to the pump used as boundary conditions for the larger tank model.

This separation of the problem into logical pieces means that the tank mixing analysis will be accomplished in stages. First will be a combined analysis of all of the effects described in earlier sections within a region assumed to be affected only by the pump, the so-called "near- 
region" for a pump. This analysis will provide a set of boundary conditions at the edge of the near-region that can be used to drive the later models that encompass more of the tank. Therefore, it must include all of the operational parameters thought to be important, including pump rotation. The analysis will then be extended to a quarter-tank model, one in which only a single pump operates. The pump will be represented by boundary conditions taken from the near-region analysis. Continuous boundary conditions will be used at the outer edges of the $1 / 4$ sector. Finally, the analysis will be extended to a half-tank model. This will use two pumps in non symmetrical positions, still with continuous boundary conditions at the edges of the $1 / 2$ sector. Each of these tasks is described in more detail below.

4a. Combined analysis in pump near-region. With the calculational response of each individual effect characterized, the model will be extended to demonstrate the combined effects of vortex behavior, free surface jet behavior, and rotating pumps. The combined analysis will not look at the effects of the jet hitting the tank wall unless it seems appropriate based on the other analyses. The purpose of the analysis within the near-region is to determine not only the mixing characteristics within that region, but also to determine a boundary condition along a computational surface within the region (or possibly at the edge of the region) that can be used to drive the quarter- and half-tank models. In this regard, this analysis is the precursor to the extended tank mixing analysis. It will include parametric variations such as viscosity, fluid property models, and turbulence models. Operational parameters that must be investigated for overall tank behavior will have to be included in the boundary conditions provided by this near-region analysis. These would include tank volume (or liquid level), pump speed and pump rotational speed, and perhaps pump elevation. Since the near-region problem is to be treated as independent of the rest of the tank, each unique set of parameters will require a separate calculation to generate the appropriate boundary conditions for the tank analysis.

4b. Quarter-tank analysis. The quarter-tank analysis will be the first step toward assessing mixing in the tank as a whole. Parametric variations will include those used earlier: viscosity, property models, and turbulence models. The effects of operational parameters, pump rotation, tank liquid level, pump speed, etc., will be included in the boundary conditions provided from the near-region analysis. Wall effects will be included in this analysis because of the model geometry.

An important part of the quarter-tank analysis will be a determination that the problem can be separated into a near-region and a quarter-tank calculation as described. This will require a demonstration that the near-region analysis is not sensitive to changing boundary conditions provided through feedback from the quarter-tank analysis. This demonstration will be attempted by completing a few external interations between the two models. Our current hope is that a combined, coupled analysis will not be needed, since such a calculation would 
be difficult to set up and very time consuming to run. Nonetheless, the demonstration that the problem can be decoupled as described is important.

4c. Half-tank analysis. The half-tank analysis will be similar to the quartertank analysis except that two pumps will be used, one in each modeled quarter. They will be in non symmetrical positions representative of the actual position in the waste tanks. The analytical effort will be similar to the quarter-tank effort, except that computational run time is expected to be greater because of the bigger model.

\section{Validation}

Code validation is an integral part of the development work described here. As each capability is developed, validation data will be sought in both the SRS literature and the open literature. Certain outside data sources have already been identified, including PNL slurry settling experiments and the British Hydromechanics Research (BHR) Group. Access to both of these data sources will require contractual agreements with the respective organizations, so neither can be considered currently available data.

In lieu of adequate data for validation, comparisons between independent calculational codes can be used. We anticipate that data will be difficult to obtain, so the present development plan includes the use of both FLUENT and CFDS-FLOW3D. While many of their respective capabilities are similar, the development and implementation of the two codes has been independent. Therefore, the use of these two codes to calculate the same problems will provide independent validation for each. In addition, the licensing agreements with both code vendors are such that there is no significant difference in cost between using one code or two. The advantage of having two codes to compare with each other outweighs the small difference in cost and establishes an ability to validate our results in an environment where adequate data are not available.

All of the documentation described in the schedule below will include computational results as well as the validation analyses discussed here. The preferred validation method will always be comparisons with data whenever appropriate data can be obtained. 
SRT-EMS-950006

Schedule and Deliverables

Task No.

Description

Preliminary draft report -

Duration to complete

1a Submerged free jet in water

Completed

1b. Wall jet in water, with extension to other property

$10 \mathrm{mw}$

models

2. Vortex analysis in water, with rotating jet

$12 \mathrm{mw}$

boundary conditions

1c. Surface jet in water

$6 \mathrm{mw}$

3. Wall climbing

$3 \mathrm{mw}$

4a. Combined analysis in pump near-region

$12 \mathrm{mw}$

$4 \mathrm{~b}$

Quarter-tank analysis

$13 \mathrm{mw}$

$4 c$.

Half-tank analysis

$16 \mathrm{mw}$

A caution with the above estimates is that the work is new development, with little to draw on from the literature once we move past applications in water. Several of our literature references are from the last few years, and documented work with non Newtonian fluids is rare. It is quite possible that as we move beyond the first task, we will have learned that the work is more difficult than originally anticipated, and that the estimates of task duration will have to be revised. This is especially true for task 4 , which relies heavily on all of the preceding work.

Deliverables for the tasks will be the preliminary draft reports listed above, final versions of the reports based on technical reviews, and detailed monthly reports documenting progress and problemis as they develop. It is anticipated that a significant number of papers for presentation at meetings and submittal to journals will come directly from these technical reports. 